\title{
Les PME axées sur la durabilité et à forte croissance : Une approche par les paradoxes 1
}

\author{
Ce document est la version « manuscrit auteur » d'un article publié dans \\ Revue Internationale PME, vol. 28 n³-4, p. 195-223 \\ Céline BÉRARD ${ }^{2}$ \\ Maître de Conférences \\ Université Lyon 2, Laboratoire COACTIS (EA 4161) \\ ISH - 14-16 av. Berthelot, 69363 Lyon Cedex 07, France \\ Celine.berard@univ-lyon2.fr
}

\author{
Christelle BRUYÈRE ${ }^{3}$ \\ Maître de Conférences \\ Université Jean Monnet de Saint-Etienne, Laboratoire COACTIS (EA 4161) \\ 6 rue Basse des Rives, 42023 Saint-Etienne Cedex 02, France \\ Christelle.bruyere@univ-st-etienne.fr
}

Séverine SALEILLES ${ }^{4}$

Maître de Conférences

Université Lyon 1, Laboratoire SAF (EA 2429)

50 avenue Tony Garnier, 69007 Lyon France

Severine.saleilles@univ-lyon1.fr

\footnotetext{
${ }^{1}$ Remerciements : ce travail a été est soutenu par l'Agence Nationale de la Recherche (ANR), dans le cadre de l'AAP «Entreprises et formes d'organisation économiques - Enjeux, mutations et permanences» (Projet Hypercrois n ${ }^{\circ}$ ANR-07-ENTR-008).

${ }^{2}$ Céline Bérard est maitre de conférences à l'Université Lyon 2, et est membre du laboratoire COACTIS. Elle détient un doctorat en Sciences de Gestion de l'Université Paris-Dauphine, et un Ph.D. en Administration des affaires de l'Université du Québec à Montréal. Ses récents travaux de recherche s'articulent autour des dynamiques de développement des PME. En particulier, ils portent sur les stratégies de croissance des PME et la gestion des risques.

${ }^{3}$ Maître de conférences à l'Université de Saint-Etienne et membre titulaire du laboratoire de recherche en gestion COACTIS, Christelle Bruyère est titulaire d'un doctorat en Sciences de Gestion de l'université d'AixMarseille 2. Ses centres d'intérêts en recherche portent sur les stratégies collectives d'accès aux soins en France, en particulier sur le management paradoxal des réseaux de santé.

${ }^{4}$ Séverine Saleilles est maître de conférences à l'Université Lyon 1, et est titulaire d'un doctorat en Sciences de Gestion de 1'Université de Montpellier 1. Elle est membre titulaire du Laboratoire de Sciences Actuarielle et Financière de l'Université Lyon 1, et membre associé de COACTIS. Ses intérêts de recherche portent sur le réseautage entrepreneurial, les stratégies de croissance et coopération des organisations à buts non-économiques, ainsi que l'institutionnalisation d'un champ organisationnel (circuits courts alimentaires).
} 


\section{Les PME axées sur la durabilité et à forte croissance : Une approche par les paradoxes}

Cet article vise à explorer les tensions paradoxales présentes dans les PME axées sur la durabilité et à forte croissance, ainsi que les pratiques mises en ouvre pour faire face à ces tensions. La recherche repose sur l'étude d'un cas unique, une PME française pionnière du secteur bio. L'analyse de ce cas permet d'identifier quatre tensions paradoxales majeures, qui s'intensifient de manière progressive durant les phases de forte croissance : identité normative et utilitariste, croissance interne et externe, exploration et exploitation, désorganisation et structuration. Elle permet de plus de repérer un ensemble de clés stratégiques, qui ont permis aux situations paradoxales de trouver leur équilibre dans des logiques de dialogue et de différenciation.

Mots-clés : Croissance - Développement durable - Paradoxe - PME - Étude de cas.

\section{Sustainability-driven and high-growth SMEs: A paradox approach}

This paper aims to explore the paradoxical tensions that are present in sustainability-driven and high-growth SMEs, and the practices implemented to deal with such tensions. This research is based on a single-case study within a French SME, a pioneer in the organic sector. The study findings reveal four major paradoxical tensions that progressively deepen in the course of high-growth periods: normative and utilitarian identities, organic and external growth, exploration and exploitation, disorganization and structuring. They also suggest a set of strategic keys, allowing to manage paradoxical situations through the perspectives of dialogue and differentiation.

Keywords: Growth - Sustainable development - Paradox - SME - Case study.

\section{Las PYME de sostenibilidad impulsada y el alto crecimiento: Un enfoque por las paradojas}

Este artículo tiene como objetivo de explorar las tensiones paradójicas que existen en las PYME de sostenibilidad impulsada y de alto crecimiento, y las prácticas implementadas para hacer frente a estas tensiones. Esta investigación se basa en un estudio de caso único dentro de una PYME francesa, pionera en el sector orgánico. El análisis de este caso identifica cuatro principales tensiones paradójicas, que intensifica gradualmente durante las fases de alto crecimiento: la identidad normativa y utilitaria, el crecimiento interno y externo, la exploración y la explotación, la desorganización y estructuración. También sugieren un conjunto de claves estratégicas para ayudar a manejar las situaciones paradójicas a través de las perspectivas de diálogo y diferenciación.

Palabras clave: Crecimiento - Desarrollo Sostenible - Paradoja - PYME-Estudio de caso. 


\section{Introduction}

Le développement durable est devenu l'une des préoccupations majeures de la société d'aujourd'hui (Shepherd et Patzelt, 2010), et il est désormais admis la nécessité de réduire les impacts néfastes, tant environnementaux que sociétaux, engendrés par des pratiques d'affaires « non durables » (Hall, Daneke et Lenox, 2010). Durant les dernières décennies, un nombre croissant de travaux s'est ainsi intéressé à «l'application des valeurs du développement durable incitant les dirigeants à prendre en considération les retombées économiques, sociales et environnementales de leurs actions »(Paradas, 2011, p. 129). Néanmoins, et comme le regrettent de nombreux auteurs, cette littérature abondante concerne surtout les grandes entreprises (Fassin, Rossem et Buelens, 2011; Kechiche et Soparnot, 2012; Paradas, 2011): les études qui portent sur les pratiques de management durable au sein des PME demeurent, quant à elles, encore limitées (Moore et Spence, 2006; Stewart et Gapp, 2014).

Pourtant, le management durable peut prendre forme au sein d'une diversité d'entreprises ; des petites comme des grandes, des nouvelles comme des anciennes. Par exemple, Hockerts et Wüstenhagen (2010) opposent les "greening Goliath" (des entreprises de grande taille et souvent relativement âgées, dont le but premier est de faire des profits) aux "David" militants. Ces derniers, de plus petite taille, peuvent être qualifiés de PME axées sur la durabilité (Parrish, 2010), en ce sens qu'il s'agit de PME qui cherchent avant tout à contribuer au bienêtre écologique et social (Hockerts et Wüstenhagen, 2010), étant moins guidées par des incitations pécuniaires que par un ensemble de normes et valeurs (Hall, Daneke et Lenox, 2010). Si ces "David" militants agissent à leurs débuts tels des «bricoleurs sociaux » (Zahra, Gedajlovic, Neubaum et Shulman, 2009), certains adoptent toutefois par la suite des pratiques d'affaires plus professionnelles et plus proches de celles des "Goliath", pour croître de manière proactive. Ces PME axées sur la durabilité qui font le choix de la forte croissance sont alors vues comme des acteurs clés de la transformation d'une industrie vers le développement durable (Hockerts et Wüstenhagen, 2010). Pour autant, peu d'études se sont intéressées à la croissance de ces entreprises (Nazarkina, 2012). Or, si la forte croissance est un moyen pour elles d'améliorer leur impact social et environnemental et, ultimement, de transformer les standards d'une industrie, elle peut aussi les amener à renier leurs valeurs et principes (Parrish, 2010): elle risque simultanément de compromettre l'impact qu'elles recherchent (Smith, Gonin et Besharov, 2013). Aussi, pour ces entreprises, la croissance représenterait en elle-même une «tension paradoxale » (Smith, Gonin et Besharov, 2013; Weinberg, 1998).

Plus généralement, la forte croissance appellerait, en effet, à une perturbation permanente des conditions d'action des PME et nécessiterait la maîtrise par ces dernières de multiples tensions pouvant être qualifiées de paradoxales (Chanut-Guieu et Tannery, 2009). Les paradoxes étant vus comme étant tour à tour conséquences et moteurs de la forte croissance (Chanut-Guieu et Guieu, 2011a), la dynamique de forte croissance des PME reposerait sur une capacité à la gestion paradoxale (Chanut-Guieu et Tannery, 2009; Grimand, 2013). Une telle capacité traduit l'aptitude à concilier des contraintes a priori contradictoires, autrement dit, à combiner - plutôt qu'à dichotomiser - des pôles opposés pour éviter de s'enfermer dans le paradoxe (Josserand et Perret, 2003). Comme le soulignent Josserand et Perret (2003, p. 165), "préconiser une gestion paradoxale [...] est cependant insuffisant si l'on n'entre pas dans une réflexion sur sa mise en æuvre ».

Dans la lignée des travaux qui allient paradoxe et croissance, la présente recherche propose ainsi d'analyser les tensions paradoxales présentes dans les PME axées sur la durabilité et à 
forte croissance, ainsi que les pratiques mises en œuvre pour y faire face dans le cadre d'une gestion paradoxale. Plus précisément, nous nous inscrivons ici dans une "perspective de la pratique » au sens défini par Rouleau, Allard-Poesi et Warnier (2007), visant à « comprendre comment les individus réalisent leurs actions en contexte, étant entendu que ces actions [...] $s$ 'inscrivent dans un contexte de relations sociales, de significations, de règles et de routines, de savoir-faire et d'objectifs donnant sens à l'action; autrement dit que ces actions actualisent et renouvellent un ensemble de pratiques existantes » (p. 17).

En premier lieu, cet article met en exergue la pertinence de mobiliser le concept de paradoxe pour étudier le phénomène de forte croissance dans les PME axées sur la durabilité. Puis, est expliquée la méthode de recherche suivie, laquelle repose sur une étude de cas réalisée auprès d'une PME française pionnière du secteur bio. Les tensions paradoxales et les pratiques repérées dans ce cas sont ensuite présentées et discutées, avant de conclure sur les implications managériales, limites et voies de recherche futures.

\section{Vers une vision paradoxale des PME axées sur la durabilité et à forte croissance}

La croissance des PME axées sur la durabilité soulève des contradictions auxquelles sont confrontés les acteurs organisationnels. En conséquence, et après avoir précisé le contexte particulier de ces entreprises, nous proposons une approche par les paradoxes pour appréhender ces contradictions et tenter de comprendre comment les acteurs peuvent apprendre à « vivre avec».

\subsection{La croissance comme source de tensions paradoxales dans les PME axées sur la durabilité}

Pour Jenkins (2009, p. 22), la durabilité renvoie à « l'objectif ultime de maintenir indéfiniment la viabilité de nos économies, les sociétés dans lesquelles elles s'inscrivent et l'environnement physique dont elles dépendent ». Les entreprises qui s'engagent dans la voie de la durabilité cherchent ainsi à répondre à divers impératifs, qu'ils soient d'ordre politique, législatif, économique, éthique, ou encore, planétaire (Berger-Douce, 2005). D'un point de vue managérial, il s'agit pour l'entreprise de prendre en considération les impacts économiques, sociaux et environnementaux de ses activités (Kechiche et Soparnot, 2012; Paradas, 2011). La plupart des études antérieures s'inscrivent néanmoins dans une logique instrumentale où la dimension économique primerait sur les dimensions sociales et environnementales, postulant que ces deux dernières dimensions puissent renforcer les gains financiers. Pour autant, les questions sociales et environnementales ne contribuent pas toujours à celles d'ordre économique (Hahn, Pinkse, Preuss et Figge, 2015) et la littérature suggère ainsi que les dirigeants et managers puissent être confrontés à des tensions et conflits face aux différentes dimensions du développement durable (Hahn, Preuss, Pinkse et Figge, 2015). Des auteurs utilisent même le terme de "paradoxes » pour appréhender les standards ou pratiques de management durable (de Colle, Henriques et Sarasvathy, 2014; Grimand, VandangeonDerumez et Schafer, 2014).

En particulier, le manque structurel de ressources conduirait souvent les PME à voir les questions de développement durable comme une contrainte, plutôt que comme une opportunité d'affaires (Courrent, 2012). En effet, les entreprises de petite taille se heurtent à certains obstacles qui risquent de les freiner dans l'adoption de pratiques durables dont, certes, 
le manque de ressources, mais aussi le manque de temps, d'incitatifs et de soutien aux initiatives durables (Bonneveux, Calmé et Soparnot, 2012). Pour autant, certaines d'entre elles mettent avant tout l'accent, non pas sur l'aspect économique, mais sur les aspects sociaux et environnementaux. Pour qualifier ces entreprises, qui voient principalement leur activité commerciale comme un moyen de contribuer au bien-être écologique et social, Parrish (2010) propose le terme d'entreprises axées sur la durabilité ("sustainability-driven"). Si leurs motivations premières s'éloignent ainsi de celles des entreprises plus traditionnelles, elles tendent aussi à adopter des pratiques d'affaires qui leur sont spécifiques (Choi et Gray, 2008). Notamment, ces entreprises se distingueraient par leur vision particulière de la croissance : la croissance ne serait pas envisagée sous l'angle de retombées financières, ni sous sa dimension idéologique, mais plutôt en termes de capacité à augmenter les retombées positives sociales et environnementales (Parrish, 2010).

Une étude menée par Hockerts et Wüstenhagen (2010) peut apporter un éclairage intéressant sur cette question de la croissance dans les PME axées sur la durabilité. En effet, en mettant en exergue les rôles complémentaires que jouent les "David" militants (des pionniers du développement durable) et les "greening Goliath" (des grandes entreprises moins ambitieuses dans leurs objectifs sociaux et environnementaux) dans la transformation durable d'une industrie, ces auteurs expliquent l'émergence de PME axées sur la durabilité et à forte croissance : les "high-growth David". Selon eux, ces entreprises sont des "David" qui "ne partagent plus la devise 'small is beautiful' des pionniers. Au contraire, ils visent clairement une croissance profitable pour étendre le marché et le défendre vis-à-vis des grands du secteur » (p. 489). Les "high-growth David" choisissent ainsi de croître de manière proactive, pour contrer une reprise en main du marché par des "Goliath" moins axés sur la durabilité. Pour grandir, ces PME tendent à combiner le meilleur des deux mondes des "David" et des "Goliath" : notamment, si elles continuent à mettre l'accent sur l'innovation de produit (force des "David"), elles visent aussi l'innovation de processus (force des "Goliath"); ou encore, si elles possèdent en tant que "David" une très bonne compréhension du marché, elles cherchent désormais à l'étendre grâce à un management plus professionnel (plus proche des "Goliath") et à une vision moins idéaliste (Hockerts et Wüstenhagen, 2010). Ainsi, alors que la littérature suggère que les PME axées sur la durabilité adoptent des pratiques d'affaires bien spécifiques (Choi et Gray, 2008), la forte croissance peut, au contraire, les amener à intégrer des pratiques plus proches de celles des entreprises dont le but premier est de faire des profits.

Pour les entreprises axées sur la durabilité, la croissance représenterait en elle-même un paradoxe (Smith, Gonin et Besharov, 2013; Weinberg, 1998). En effet, alors que ces entreprises cherchent à croître pour améliorer leur impact social et environnemental (Parrish, 2010), la croissance peut simultanément représenter une menace à l'égard de cet impact recherché (Smith, Gonin et Besharov, 2013). De manière "paradoxale », ces PME qui, par leur croissance, peuvent contribuer à l'évolution des pratiques de leurs concurrents vers le développement durable (Hockerts et Wüstenhagen, 2010; Weinberg, 1998), auraient du mal à grandir tout en restant axées sur la durabilité (Weinberg, 1998). Pour Smith, Gonin et Besharov (2013), cela s'expliquerait notamment par le fait que si des facteurs peuvent contribuer aux «missions sociales » dans les petites organisations (en particulier, des facteurs tels que l'ancrage local, la construction d'une confiance partagée, et l'empreinte des valeurs et de la morale des fondateurs), la contribution de ces mêmes facteurs diminue dès lors que la taille augmente.

Plus généralement, la forte croissance implique en effet une altération drastique, car non progressive, dans l'évolution d'une PME. Elle est associée à un important changement dans 
ses stratégies, actions et comportements (Moreno et Casillas, 2007), et place son dirigeant dans une situation très complexe qui, de plus, évolue très rapidement (Séville et Wirtz, 2010). Comme le résument Chanut-Guieu et Tannery (2009), la forte croissance revient à vivre et à créer continuellement des perturbations et des déséquilibres radicaux. Ces auteurs soulignent ainsi la nécessité « de recourir à un cadre théorique qui traite directement de la multitude des tensions qui parcourent l'entreprise et l'organisation » (p. 7) et, en particulier, l'intérêt de s'appuyer sur des approches mobilisant le concept de "paradoxe". Dans cette perspective, la réussite des stratégies de forte croissance des PME reposerait sur la gestion combinée de couples paradoxaux tels que, selon Chanut-Guieu et Guieu (2011b): l'intention et l'adaptation (la volonté de suivre son intuition et la nécessité de s'adapter à son environnement), l'exploration et l'exploitation (l'exploitation de connaissances existantes et l'exploration de nouveaux horizons), la réalisation d'opérations de croissance interne et externe (grandir de l'intérieur en s'auto-développant et grandir en procédant à des fusionsacquisitions, en développant de fortes relations avec ses partenaires).

\subsection{Les paradoxes et les logiques organisationnelles paradoxales}

Les recherches qui mobilisent une lecture paradoxale étudient la façon dont les organisations considèrent et gèrent les demandes contradictoires et conflictuelles qui émergent dans divers domaines de gestion, tels que l'innovation, la gestion des ressources humaines, la gestion du changement, etc. (Chanut-Guieu, Guieu, Tannery et Dana, 2014). Les travaux fondateurs de Martinet (1990) identifient d'ores et déjà bons nombres de paradoxes organisationnels : délibéré/émergent, autonomie/interdépendance, potentialisation/actualisation, etc. Le concept de paradoxe traduit donc la présence simultanée de deux états a priori incompatibles, comme le sont par exemple la coopération et la compétition, ou encore, l'innovation et l'efficience (Eisenhardt, 2000). Plus précisément, un paradoxe désigne des "éléments contradictoires mais reliés entre eux - éléments qui semblent logiques pris isolément, mais absurdes et irrationnels lorsqu'ils surviennent simultanément »(Lewis, 2000, p. 760).

La nature même du paradoxe interdit de le réduire au dilemme. Le paradoxe dans ce cadre n'a plus à être ignoré, ni même à être dépassé (à travers la recherche d'un compromis, d'une synthèse bien souvent illusoire et réductrice), mais doit être reconnu pour ce qu'il est : un élément moteur des dynamiques organisationnelles et une source potentielle d'apprentissage. Aussi, Smith et Lewis (2011) notent qu'un nombre croissant de chercheurs mobilise cette perspective du paradoxe pour analyser des phénomènes organisationnels complexes. Selon ces auteurs, une telle perspective offre une vision alternative aux tensions auxquelles font face les organisations : elle reconnait que ces dernières doivent, pour être performantes sur le long terme, répondre simultanément à, et non pas choisir entre, des demandes ou contraintes contradictoires. L'approche par le paradoxe envisage ainsi la contradiction sous un angle positif comme un moyen de réguler des éléments contradictoires présents au sein des organisations. La vision paradoxale représente une occasion de changement, pour l'individu comme pour l'organisation, en encourageant une dynamique créative (Fayard et Blondeau, 2014). Dès lors, cette approche permet de comprendre que les tensions des PME axées sur la durabilité, exacerbées par la croissance, ne sont pas pathologiques, mais bien génératrices de bricolages ingénieux qui permettent de «vivre avec». La gestion de cette cohabitation permet, en effet, d'exploiter les tensions de manière créative en capturant les avantages de chacun des deux pôles du paradoxe (Eisenhardt, 2000). Le paradoxe devient générateur d'une troisième voie créatrice de valeur. 
En particulier, les travaux de Josserand et Perret (2003) permettent de recenser trois grandes logiques organisationnelles paradoxales qui offrent une issue positive aux paradoxes: les logiques de différenciation, de dialogue et de disparition. Premièrement, la logique de différenciation s'exprime dans l'affirmation des différences entre les pôles d'un paradoxe. Elle consiste à clarifier chacun des pôles pour ensuite affirmer leurs spécificités. Le paradoxe y est non pas tranché, mais simplement parcellisé. Cette logique ne donne pour autant pas lieu à deux stratégies " simples ", dans la mesure où elle reconnaît la complémentarité des contradictions en présence. Elle repose sur un travail de segmentation en limitant les points de rencontres entre les pôles distants pour éviter les frictions. Deuxièmement, la logique de dialogue vise à gérer le paradoxe en favorisant les échanges entre les pôles pour créer des interactions réciproques continues, non pas dans le but de produire une voie moyenne mais dans celui de permettre un enrichissement mutuel. Il en est ainsi de l'eau tiède qui n'est ni chaude, ni froide et qui a été obtenue en excluant la potentialité de chacun des extrêmes : "Alterner le chaud et le froid est une stratégie, asperger avec de l'eau tiède n'est pour nous qu'un refus stratégique » (Bernard-Weil, 2002, p. 9). Troisièmement, la logique de disparition consiste à gérer le paradoxe en cherchant à le faire disparaître. Elle ne nie pas l'existence du paradoxe, dans la mesure où elle consiste à le reconnaître pour ensuite le supprimer. Il s'agit d'amener les acteurs à se représenter la réalité différemment de manière à ce que le paradoxe tombe de lui-même. Tout l'enjeu de cette logique est de sortir les acteurs de leurs cadres de références par un saut cognitif. En conclusion, il apparait que l'ensemble de ces logiques organisationnelles relève d'une représentation paradoxale, qui reconnait l'existence des paradoxes sans chercher à choisir : les logiques de différenciation et de dialogue consistent à gérer le paradoxe en permettant l'expression des dimensions contraires, tandis que la logique de disparition vise à faire «tomber » le paradoxe pour libérer l'organisation de son emprise. Cette grille de lecture paradoxale peut alors constituer un outillage conceptuel intéressant pour appréhender les tensions paradoxales des PME axées sur la durabilité et à forte croissance.

Dans cette perspective, et en nous appuyant sur l'étude de cas d'une PME axée sur la durabilité, nous cherchons à mettre en relief les contradictions que suscite sa forte croissance. Précisons toutefois que le concept de paradoxe est ici exploité dans sa capacité heuristique à faire émerger du sens (Perret et Josserand, 2003) : les tensions paradoxales explorées dans la présente recherche relèvent d'une construction mentale du chercheur, qui se sert du paradoxe comme d'un outil conceptuel pour se représenter une réalité complexe.

\section{L'étude d'un pionnier du bio : le cas de la PME «BioDistri »}

Cette partie présente la méthode de recherche suivie, qui repose sur l'étude d'une PME axée sur la durabilité et à forte croissance. Elle décrit également la trajectoire de croissance de la PME étudiée, citée dans le présent article sous le nom fictif de « BioDistri ».

\subsection{La méthode de collecte et d'analyse des données}

La méthodologie retenue est l'étude de cas unique (Yin, 1994), l'objectif étant d'explorer des pratiques ou des phénomènes nouveaux (Wacheux, 1996). Le cas choisi est exemplaire tant sur le plan de la démarche guidée par le développement durable, que sur celui de la forte croissance (cf. Figure 1). D'une part, il s'agit d'une entreprise pionnière du bio, qui a contribué au développement de la filière bio en France et à l'étranger dans une logique de commerce équitable, militantisme revendiqué du fondateur. Cette PME a également 
développé une réelle politique sociale basée sur la diversité et l'évolution interne. D'autre part, grâce à une stratégie de croissance volontariste, elle a vécu une croissance pouvant être qualifiée de forte, étant donné qu'elle est passée, en quelques années, d'un effectif d'une quarantaine de salariés à plus de 200 , et a enchainé des taux de croissance annuel du chiffre d'affaires de plus de $25 \%$. Ainsi, et bien que les PME à forte croissance aient fait l'objet de définitions diverses et variées dans les travaux antérieurs, BioDistri respecte largement celles proposées par nombre d'auteurs qui associent les PME à forte croissance à des entreprises ayant une croissance annuelle d'au moins 20\% sur une période de cinq ans (Fisher, Reuber, Habadou, Johnson et Lee, 1997) ou de quatre ans (Nicholls-Nixon, 2005), par exemple. En outre, c'est au terme de cette phase de forte croissance que nous avons eu l'opportunité d'explorer la façon dont les acteurs de cette aventure l'ont vécue, de repérer les tensions paradoxales qui sont apparues et d'analyser la façon dont ces acteurs s'en sont emparés.

Figure 1 - La croissance en CA et en effectif de BioDistri

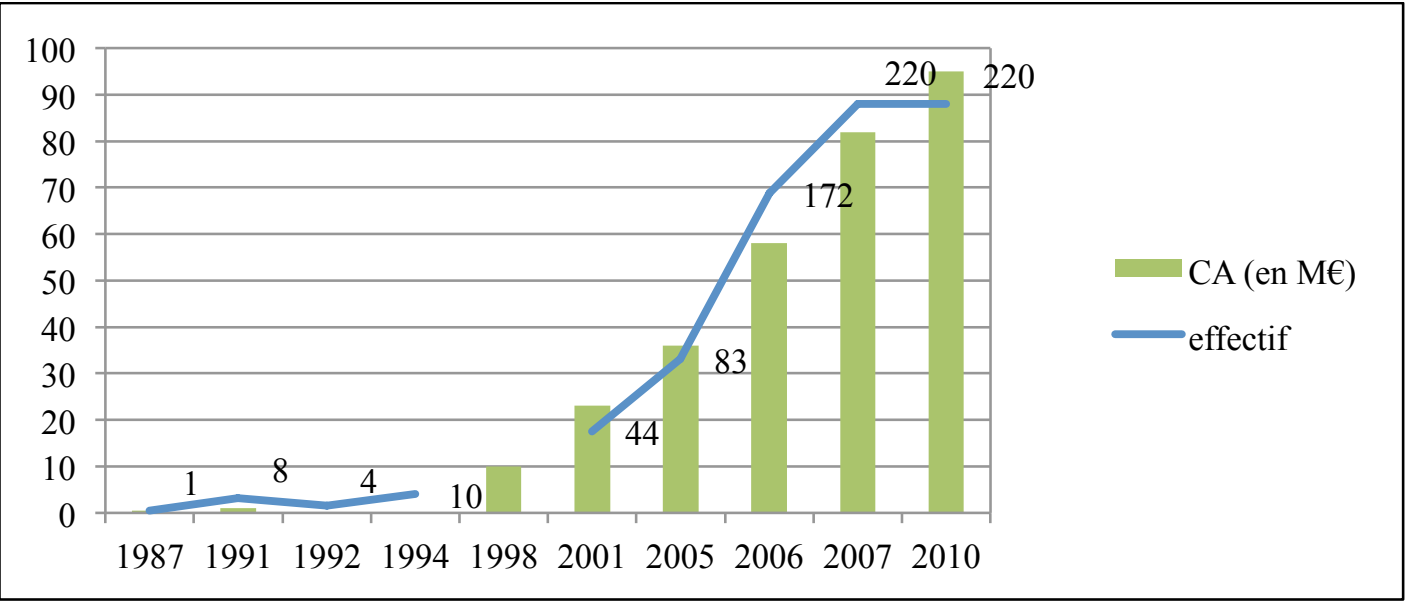

Des données secondaires (dossiers de presse, articles de presse, entretiens vidéo et radio du dirigeant) ont été croisées avec des données primaires collectées dans le cadre d'un projet de recherche collectif. Une visite du site et des entretiens individuels semi-directifs centrés (d'une durée comprise entre $1 \mathrm{~h}$ et $2 \mathrm{~h} 30$ ) ont été réalisés avec le fondateur de la PME et six responsables de fonction dans l'entreprise (cf. Tableau 1), dont cinq membres du comité de direction. La plupart des enquêtés ont connu l'intégralité de la phase de forte croissance (et deux d'entre eux étaient, de plus, présents depuis la création de l'entreprise). Pour autant, nous avons aussi voulu connaitre le regard de nouveaux arrivés comme la responsable logistique. Le guide d'entretien s'attachait essentiellement aux faits et aux comportements : récit de la vie de l'entreprise et de son marché (en insistant sur les événements clés), parcours de l'enquêté et de la fonction qu'il occupe. La fin de l'entretien permettait également au répondant de s'exprimer sur les difficultés rencontrées, les éléments permettant d'expliquer le succès et sa vision de l'évolution de cette entreprise. Les entretiens ont été enregistrés et intégralement retranscrits, pour un volume total de 148 pages.

Tableau 1 - Présentation des interviewés

\begin{tabular}{lllll}
\hline Id. & Fonction actuelle & Sexe & $\begin{array}{l}\text { Date } \\
\text { d'entrée }\end{array}$ & $\begin{array}{l}\text { Fonctions précédemment exercées dans } \\
\text { l'entreprise }\end{array}$ \\
\hline E1 & Président & H & 1987 & Fondateur, PDG \\
\hline E2 & Secrétaire général & F & 1996 & $\begin{array}{l}\text { Assistante de direction, responsable RH - } \\
\text { communication }\end{array}$ \\
\hline E3 & Développeur nouvelles filières & H & 1989 & $\begin{array}{l}\text { Développement marché magasins, sourcing (pas } \\
\text { de poste défini) }\end{array}$ \\
\hline
\end{tabular}




\begin{tabular}{lllll}
\hline E4 & Responsable contrôle de gestion & H & 2002 & Comptabilité-finance-contrôle \\
\hline E5 & Responsable logistique & F & 2009 & N.C. \\
\hline E6 & Responsable qualité & F & 1998 & $\begin{array}{l}\text { Stagiaire, responsable de quai, responsable } \\
\text { qualité services et produits }\end{array}$ \\
\hline E7 & DRH & H & 2003 & Adjoint RH \\
\hline
\end{tabular}

Ensuite, l'analyse des données a été réalisée en deux temps. En premier lieu, un relevé d'évènements (Miles et Huberman, 2003) a été établi pour dresser la trajectoire de croissance de la PME. Afin d'améliorer la validité descriptive et limiter les biais liés aux entretiens rétrospectifs, les informations collectées lors des entretiens ont été croisées avec les données secondaires. En second lieu, un codage ouvert des transcriptions d'entretiens a été effectué pour faire émerger les tensions paradoxales, ainsi que les pratiques ou actions entreprises pour y faire face. Pour s'assurer de la fiabilité de la démarche, deux des entretiens (ceux effectués auprès du Président et du Secrétaire général) ont fait l'objet d'un double-codage.

\subsection{La trajectoire de croissance de BioDistri}

BioDistri, une entreprise française, a été fondée en 1987 par un maraicher "atypique ». Musicien de formation, parisien d'origine, il a effectué son "retour à la terre » dans les années 80 pour reprendre l'exploitation d'un oncle pionnier de l'agriculture biologique. L'activité de son entreprise était alors orientée vers la commercialisation de fruits et légumes bio de la Provence vers des grossistes allemands. L'entreprise s'est rapidement distinguée sur un marché embryonnaire et peu structuré. Alors que la croissance était à ses débuts portée par celle du marché allemand, un événement a remis en cause ce modèle. "Gros pépin économique [...] choc » (E1), «catastrophe» (E3), «moment tellement pénible » (E2), les mots ne manquent pas pour qualifier la grève des transports de 1992 et l'«espèce d'électrochoc » (E1) qu'elle a provoqué dans l'histoire de l'entreprise et son évolution. Les clients allemands se sont tournés vers l'Italie, 4 des 8 salariés de l'époque ont été licenciés et la diversification de l'amont (d'abord en Méditerranée, puis plus loin) comme de l'aval (marché français, magasins spécialisés) a été édifiée en logique clé du développement de BioDistri.

Si, sauf en 1992-93, l'entreprise a jusqu'alors «cru, mais sans s'en rendre compte ou sans le vouloir » (E1), la décision a été prise en 1994, lors d'un séminaire collectif de travail, d'aller vers une croissance volontariste. La PME entre alors dans un cycle de croissance soutenue, puis opère en 2001 un virage stratégique en décidant de servir la grande distribution, vue comme "un relais de croissance de la demande important» (E1) et un moyen de convertir davantage de consommateurs à la bio. Face à l'arrivée de la GMS sur le marché bio suite aux multiples crises sanitaires de la fin des années 90, BioDistri se fixe en effet comme objectif d'occuper "une position sur le marché qui soit suffisamment importante pour essayer de continuer à construire un marché bio assez indépendant du marché conventionnel »(E1). Le marché devient beaucoup plus concurrentiel avec de nombreux nouveaux entrants non spécialistes du bio (distributeurs conventionnels ou coopératives agricoles) capables de fournir de gros volumes. Cette «très grande ouverture très rapide » (E2) du marché bio, dopée par une incitation forte des pouvoirs publics et par le fait que «la bio apparaît comme une solution également économique » (E2) marque à partir de 2008-2009 une nouvelle phase de développement pour BioDistri, dont "le marché est en train de changer»(E2). La croissance se ralentit pour " s'asseoir sur la courbe de croissance du marché » (E4). Certains clients, notamment en GMS, se tournent vers les nouveaux entrants. Plusieurs autres grossistes bios multi-produits «moins bien placés qu'une grosse coopérative subventionnée qui est spécialisée sur 3 ou 4 produits» (E2) rencontrent des difficultés. Plusieurs enseignes décident «de ne plus mettre du tout des produits étrangers dans leurs rayons » (E3). Le choix 
est alors fait de privilégier le «cour de métier» (E2) de BioDistri depuis la crise de 1992, à savoir les magasins spécialisés. La proportion du débouché «magasins spécialisés » dans le chiffre d'affaires de la PME passe de $34 \%$ en 2007 à $41 \%$ en 2010, et la France devient le marché majoritaire (76\% du CA en 2010 contre $48 \%$ en 2007$)$.

Le tableau 2 résume les évènements clés qui jalonnent sa trajectoire de croissance.

Tableau 2 - Événements clés de la trajectoire de croissance de BioDistri

\begin{tabular}{|c|c|c|}
\hline $\begin{array}{l}\text { Phases de } \\
\text { croissance }\end{array}$ & Événements clés de la vie de l'entreprise & Événements de l'environnement \\
\hline $\begin{array}{l}\text { 1987-1991 } \\
\text { Des débuts } \\
\text { prometteurs } \\
\text { CA } * 2 \\
\text { Effectif } * 8\end{array}$ & $\begin{array}{l}1987: \text { création d'une entreprise } \\
\text { d'exportation de fruits et légumes bio } \\
\text { provençaux vers l'Allemagne } \\
\text { Développement d'un approvisionnement en } \\
\text { Corse puis en Espagne (1989) }\end{array}$ & $\begin{array}{l}\text { Demande faible et surtout allemande } \\
\text { Offre peu structurée (production par des } \\
\text { pionniers et pas de certification ; } \\
\text { distribution réalisée par des acteurs peu } \\
\text { «professionnels ») } \\
\text { Quasiment pas de concurrence }\end{array}$ \\
\hline $\begin{array}{l}\text { 1992-1994 } \\
\text { La crise } \\
\text { CA non connu } \\
\text { Effectif divisé par } 2 \\
\text { puis } * 2,5\end{array}$ & $\begin{array}{l}1992 \text { : licenciement de } 4 \text { salariés } \\
\text { Décision de diversifier l'aval (marché des } \\
\text { magasins spécialisés) et l'amont (étranger) }\end{array}$ & $\begin{array}{l}1992 \text { : Grève des transports } \\
\text { Développement en France de magasins } \\
\text { spécialisés bio } \\
\text { Règlement européen sur l'agriculture } \\
\text { biologique (1991) et développement de } \\
\text { la production bio surtout en Italie et } \\
\text { Espagne }\end{array}$ \\
\hline $\begin{array}{l}\text { 1994-2001 } \\
\text { Croissance } \\
\text { volontariste } \\
\text { CA } * 2 \text { (entre } 1998 \\
\text { et } 2001) \\
\text { Effectif *4 }\end{array}$ & $\begin{array}{l}1994 \text { : décision collective de croissance } \\
\text { Développement de filières de production en } \\
\text { méditerranée, Afrique et Amérique } \\
\text { Développement du débouché des magasins } \\
\text { spécialisés et des marchés français et nord- } \\
\text { européens } \\
1998 \text { : Création d'une fonction qualité } \\
2001 \text { : Création d'un nouveau bâtiment HQE }\end{array}$ & $\begin{array}{l}\text { Crises sanitaires (vache folle, tremblante } \\
\text { du mouton, poulet à la dioxine, etc.) et } \\
\text { explosion de la demande de produit } \\
\text { biologiques }\end{array}$ \\
\hline $\begin{array}{l}\mathbf{2 0 0 1 - 2 0 0 5} \\
\text { Ouverture au } \\
\text { marché des GMS } \\
\text { et renforcement de } \\
\text { la croissance } \\
C A * 1,5 \\
\text { Effectif } * 2\end{array}$ & $\begin{array}{l}\text { 2002-2003 : entrée au capital puis rachat } \\
\text { d'un conditionneur pour la GMS } \\
2003 \text { : achat d'un concurrent en Bretagne } \\
\text { Développement de nouvelles filières en } \\
\text { Afrique }\end{array}$ & $\begin{array}{l}\text { Démocratisation du bio avec des } \\
\text { consommateurs plus regardants sur les } \\
\text { prix et moins sur la qualité, et l'appui } \\
\text { aux producteurs } \\
\text { Arrivée sur le marché de nouveaux } \\
\text { offreurs (grossistes conventionnels, } \\
\text { coopératives agricoles) et distributeurs } \\
\text { (GMS) }\end{array}$ \\
\hline $\begin{array}{l}\text { 2005-2008 } \\
\text { Ouverture du } \\
\text { capital et poursuite } \\
\text { de la croissance } \\
\text { soutenue } \\
C A * 2,5 \\
\text { Effectif *2,6 }\end{array}$ & $\begin{array}{l}2005 \text { : Entrée d'un fonds d'investissement (à } \\
51 \% \text { ) dans le capital } \\
\text { 2005-2006 : recrutement d'une équipe de } \\
\text { direction : DAF, DG (avec un échec), } \\
\text { directeur commercial } \\
2006: \text { achat d'un concurrent belge spécialisé } \\
\text { dans la GMS } \\
2007 \text { : achat d'un concurrent spécialiste des } \\
\text { fruits et légumes espagnols }\end{array}$ & $\begin{array}{l}\text { Grenelle de l'environnement (2007) : } \\
\text { politique publique de développement de } \\
\text { la production et de la demande bio en } \\
\text { France } \\
\text { Arrivée des industriels et de la } \\
\text { restauration hors domicile sur le marché }\end{array}$ \\
\hline $\begin{array}{l}2008-2010 \\
\text { Ralentissement de } \\
\text { la croissance et } \\
\text { retour aux } \\
\text { fondamentaux }\end{array}$ & $\begin{array}{l}\text { Développement de nouvelles filières et } \\
\text { renforcement de filières existantes en France } \\
2009 \text { : partenariat avec un distributeur } \\
\text { spécialisé en restauration hors domicile } \\
2009 \text { : lancement d'une offre de jus de fruits } \\
2009 \text { : recrutement d'un responsable de la } \\
\text { logistique inter-sites } \\
2009 \text { : ouverture d'un site parisien pour } \\
\text { servir les magasins spécialisés } \\
2010 \text { : lancement d'une offre de livraison de }\end{array}$ & $\begin{array}{l}\text { Fort développement des circuits courts } \\
\text { alimentaires, de la demande de produits } \\
\text { locaux } \\
\text { Remise en question des produits bios } \\
\text { exportés (doutes sur la certification, } \\
\text { impact environnemental, etc.) } \\
\text { Décision de plusieurs magasins } \\
\text { spécialisés de ne proposer plus que des } \\
\text { produits locaux }\end{array}$ \\
\hline
\end{tabular}




\section{Les tensions et logiques paradoxales de BioDistri}

L'analyse du cas de BioDistri a permis de dégager quatre grandes tensions paradoxales qui évoluent au fil de sa trajectoire de croissance. Elle a, de plus, permis de repérer un ensemble de " clés » stratégiques dont l'objectif n'est pas de mettre en avant une boîte à outils bien maîtrisés, mais plutôt des pratiques organisationnelles ouvrant la voie à une possible équilibration du système. En référence à la grille de lecture paradoxale de Josserand et Perret (2003), les paradoxes trouvent leur issue dans deux logiques organisationnelles majeures : les logiques de dialogue et de différenciation.

\subsection{Les tensions paradoxales dans la trajectoire de croissance de BioDistri}

Les quatre tensions paradoxales repérées dans le cas de BioDistri sont décrites ci-après, avant d'être analysées sous une logique temporelle.

\subsubsection{La tension entre identité normative et identité utilitariste}

En reprenant les termes utilisés par Moss, Short, Payne et Lumpkin (2011), on peut repérer au sein de BioDistri une identité organisationnelle normative (celle des entreprises à visée essentiellement sociale), mais aussi une identité utilitariste (identité caractéristique des entreprises «traditionnelles »). Or, et comme précédemment mentionné, les pratiques des entreprises à visée sociale diffèrent de celles à visée principalement économique. La double identité de BioDistri conduit ainsi inévitablement à des tensions au sein de l'entreprise, et ce, dès sa phase de croissance volontariste.

Cette croissance volontariste correspond, au départ, à une volonté de la PME développer l'agriculture biologique pour «transformer une agriculture qui est plutôt polluante en une agriculture qui est de bonne qualité » (E1). Notamment, son entrée sur le marché de la grande distribution est justifiée ainsi : «si on ne le fait pas, les gens du conventionnel vont petit à petit agréger des savoir-faire bio et le marché sera détourné, il changera de main »(E1). Ce mode de raisonnement est caractéristique d'une identité normative. On constate aussi que l'entreprise privilégie le respect de certaines valeurs, quitte à ce que cela implique des pratiques moins efficientes économiquement : accepter de la marchandise non-conforme car «on leur a garanti qu'on allait prendre leur récolte »(E5), bifurquer certains flux vers l'exportation pour répondre à la demande de production française des magasins spécialisés tout en conservant ses fournisseurs étrangers, etc. Le développeur de nouvelles filières parle même d'un réel "engagement moral quand on va faire faire des productions au Maroc" (E3). Pour autant, on ne peut nier qu'une autre logique s'est progressivement installée au sein de la PME. Ainsi, alors que les débuts étaient clairement ceux d'un projet purement militant, BioDistri va progressivement se doter d'une identité plus utilitariste : "En 93, je crois que c'est la première fois qu'il [le dirigeant] a commencé à avoir des préoccupations économiques, commencer à regarder d'avoir des bénéfices pour pouvoir investir justement et 
pouvoir mettre en place des nouveaux projets » (E2). A partir de là, l'entreprise entre «dans une phase où la rentabilité est devenue une préoccupation pas forcément numéro 1 mais elle est devenue importante. C'est sûr. Ce n'était pas le cas avant.»(E4). Certains choix font débat. Par exemple, l'entrée sur le marché de la grande distribution va impliquer l'embauche de personnels à forte culture commerciale et le rachat d'entreprises aux identités organisationnelles clairement utilitaristes. « La grande distribution, elle n'est pas capable de changer ses pratiques [...] même quand elle vient dans le bio »(E2).

Le fondateur de BioDistri revendique ce paradoxe entre identités normative et utilitariste. Il relate par exemple sa décision d'ouvrir le capital à des investisseurs : "Quand je fais rentrer des actionnaires financiers dans le capital, je me dis: 'on va voir ce que c'est, on va aller dans la gueule du loup'. Quand on est dans un milieu plutôt alternatif, on regarde le monde financier comme étant un monde absolument épouvantable. Seulement, il faut y aller. Je sais maintenant que c'est un monde épouvantable. Mais, tout cela est intéressant et on ne peut éviter aucun paradoxe $»(\mathrm{E} 1)$.

Cette tension entre deux identités est caractéristique des organisations qui, au départ, avaient plutôt une visée sociale et qui ont crû pour améliorer leur impact environnemental et social. Par exemple, en étudiant le cas d'organismes de microfinance, Battilana et Dorado (2010) font un constat similaire de tensions entre logique de développement social et logique bancaire. Ces entreprises dites hybrides, autrement dit, qui combinent plusieurs logiques institutionnelles, distinctes voire contradictoires (Pache et Santos, 2010), font l'objet d'un intérêt croissant dans la littérature. En effet, si la combinaison de logiques oblige ces organisations à se conformer à des normes et des demandes qui peuvent être conflictuelles, elle leur permet tout à la fois de combiner capitaux, connaissances tacites et régimes de justification. D'ailleurs, leur capacité d'innovation risquerait d'être amoindrie si une logique devenait dominante (Jay, 2013).

\subsubsection{La tension entre exploration et exploitation}

L'exploration et l'exploitation des connaissances, les deux formes d'apprentissage organisationnel identifiées par March (1991), sont simultanément présentes chez BioDistri et constituent l'une des tensions paradoxales perçues, là encore, dès sa phase de croissance volontariste.

Dès ses débuts, BioDistri se caractérise par sa capacité à expérimenter des idées radicalement nouvelles et à innover de manière avant-gardiste. Elle se distingue ainsi par ses activités d'exploration, qui conduisent à créer de nouvelles connaissances, compétences ou manières de faire, et à explorer des champs radicalement nouveaux (Bierly et Daly, 2007; March, 1991). En particulier, la PME réussit à introduire, avant ses concurrents, nombre de produits nouveaux sur le marché : "souvent l'entreprise a été une des premières à introduire une nouvelle origine par exemple, ou à faire un nouveau métier. L'exemple que je vais reprendre, c'est que l'on a été les premiers à faire de la banane bio »(E2). Elle a multiplié les expérimentations à partir d'une "intuition un peu calculée »(E3), avec parfois de lourds échecs (comme le lancement de la mangue bio du Costa-Rica dès 1995 alors que le marché n'était pas mûr). Pour autant, les échecs n'entachent en rien la volonté de BioDistri d'éprouver de nouvelles idées. En effet, « des échecs il y en a toujours, mais l'important c'est qu'il y ait plus de réussites que d'échecs. Et puis des fois c'est relancé, des fois tu es trop en avance sur un projet » (E3). 
Très rapidement, la PME met aussi l'accent sur la maîtrise et l'amélioration des technologies existantes et de la qualité. Elle cherche à améliorer l'efficience et à tirer profit des compétences et connaissances existantes, ce qui est caractéristique des entreprises qui mènent des activités d'exploitation (Bierly et Daly, 2007; March, 1991). BioDistri se distingue ainsi sur le marché en étant "très obsessionnel sur la qualité du produit, la qualité du service rendu aux clients, etc. " (E1). La responsable qualité met en avant la mise en place d'un système de traçabilité et le développement d'une " trentaine d'audits sur site, entre les audits de certification et les audits clients »(E6). La qualité logistique est également fréquemment revisitée : «comment transite la marchandise ; est-ce qu'on peut éviter des voyages inutiles? $C$ 'est la politique générale du groupe d'aller travailler avec un consultant sur la logistique et savoir comment développer la société » (E6). De plus, il existe une réelle volonté de réajuster les procédures et moyens en vue d'améliorer continuellement la situation. Ces ajustements sont en eux-mêmes des conséquences de la croissance et s'expliquent ainsi par la trajectoire suivie par BioDistri : "à l'époque, il n'y avait pas de procédures, chacun était capable de tout faire, sans tâches très prédéfinies. Donc, l'objectif de la qualité, c'était d'harmoniser les procédures des différents sites qui nous ont rejoints. C'est simple à dire mais c'était compliqué » (E6).

En définitive, si BioDistri a mis l'accent à ses débuts sur une démarche d'exploration, son obsession pour la qualité (des produits, des processus, des services) l'a rapidement amené à la conjuguer avec une démarche d'exploitation. Or, Bierly et Daly (2007) rappellent que l'exploration et l'exploitation des connaissances sont effectivement complémentaires et que leur interaction contribue à la croissance, tout en reconnaissant que ce double effort peut être source de tensions, particulièrement dans les PME qui ont des ressources limitées. Pour autant, les entreprises seraient «de plus en plus confrontées à la nécessité de combiner innovation d'exploration et innovation d'exploitation » (Chanal et Mothe, 2005, p. 189).

\subsubsection{La tension entre croissance interne et croissance externe}

Pour se développer, BioDistri a eu recours à une croissance interne et externe. Pour ChanutGuieu et Guieu (2011b), la réalisation de ces deux types d'opérations de croissance constitue d'ailleurs l'un des couples paradoxaux que peut être amenée à gérer une PME en hypercroissance, le succès d'une forte croissance étant lié, en partie, à la combinaison de toutes les modalités de croissance (croissance organique, fusions et acquisitions, partenariats et alliances).

Tout au long de sa trajectoire de croissance, BioDistri s'est développé en réalisant des opérations de croissance interne. De manière générale, "à chaque fois qu'il y a eu une croissance, on a agrandi les locaux » (E2). Ces opérations ont donné lieu à l'agrandissement du site provençal, ou encore, à la création d'un bâtiment à Haute Qualité Environnementale. Plus récemment, la PME s'est fixée "une autre priorité, c'est d'ouvrir l'entrepôt de Paris » (E5), dédié aux magasins spécialisés. Si l'entreprise a initié, et poursuivi depuis, une stratégie de croissance interne, c'est principalement en réponse à sa stratégie volontariste de diversification. D'une part, en lien avec sa politique de diversification géographique, BioDistri a développé de nombreuses filières d'approvisionnement à l'étranger (avec, par exemple, la création d'un site au Maroc en 1996 ou au Togo en 2005). D'autre part, la diversification de la gamme (et l'innovation au sein de celle-ci) a permis de répondre à la demande croissante du marché, en ayant « un positionnement qui était de pouvoir servir tous les fruits et légumes d'un étal »(E2). 
Mais depuis le virage stratégique visant à s'ouvrir à de nouveaux marchés, BioDistri a aussi procédé à des opérations de croissance externe pour renforcer son développement. Dès lors que la PME a décidé, en 2001, de servir la grande distribution, elle a cherché à « acheter de la compétence » (E1) et cela aboutit à l'acquisition d'une entreprise de conditionnement pour la GMS. La stratégie de croissance externe s'est alors poursuivie (avec, par exemple, l'acquisition d'un concurrent belge spécialisé dans la GMS), dans l'objectif d'occuper «une position sur le marché qui soit suffisamment importante »(E1). Si les opérations de croissance externe ont principalement été motivées par l'ouverture au marché des GMS, elles ont aussi permis à la PME d'acquérir des concurrents en France (notamment en Bretagne en 2003) et à l'étranger (dont l'achat, en 2007, d'une entreprise espagnole spécialisée dans les fruits et légumes). C'est cette stratégie de croissance externe qui a conduit le dirigeant à faire entrer en 2005 un investisseur financier au capital, en raison des investissements conséquents qu'elle requiert et du besoin de disposer des compétences nécessaires pour gérer de telles opérations. Par ailleurs, chez BioDistri, ces opérations passent aussi par la mise en place de partenariats pour développer de nouveaux marchés : "L'arrivée de la restauration hors domicile qui n'était pratiquement pas présente, ça a été un facteur important. A tel point qu'on a signé un partenariat avec des gens très implantés en restauration collective »(E2).

Comme le souligne Mustar (2002), si les fusions-acquisitions jouent un rôle fondamental pour beaucoup d'entreprises en forte croissance, le recours à la seule croissance externe y est toutefois rare. Le cas de BioDistri illustre clairement ce constat, étant donné que cette PME en forte croissance a connu "une croissance de la société même, mais une croissance externe également »(E6).

\subsubsection{La tension entre désorganisation et structuration}

Le dernier paradoxe identifié au sein de BioDistri est celui de la cohabitation entre désorganisation et structuration. En effet, la PME ayant atteint un certain seuil de développement, elle a cherché à structurer son "désordre créateur » (Thiétart et Forgues, 2006). Étant donné que c'est dans l'ambiguïté des situations et des règles prescrites que se révèle l'ingéniosité des acteurs (Alter, 2002), l'équipe dirigeante s'est trouvée dans la nécessité de penser simultanément souplesse d'actions et formalisme.

Pour illustrer cette ambivalence entre le besoin d'ordre et de désordre nécessaire à la croissance d'une PME (Fombrun et Wally, 1989), le fondateur relate l'arrivée d'un nouveau directeur général : "un type extrêmement brillant, intelligent qui a commencé à structurer toute l'entreprise parfaitement. [...] Et au bout d'un an, l'entreprise était structurée. Mais elle s'est arrêtée. Cela roulait, cela marchait, tout était parfait, mais c'était fini, plus rien » (E1). D'un autre côté, l'état de crise permanent lié à la croissance implique un «besoin justement de mettre en place toute cette organisation [...] et des gens qui aient une vision globale des questions et qui les traitent toutes en même temps » (E1).

Plus précisément, quand l'entreprise comptait moins de 50 salariés, "tout le monde faisait tout. Il y avait le président et un grand râteau en-dessous» (E7). Mais dès 2004, et du fait de la forte croissance, « on a structuré les choses » (E7). Cette structuration a consisté d'abord en la création d'un niveau de management intermédiaire puis, suite au retrait progressif du fondateur et à l'entrée au capital de l'investisseur, à la création d'un comité de direction. Cette structuration traduit notamment la volonté du fondateur de mettre en place un système de gouvernance autonome. Néanmoins, celle-ci a été réalisée « à tâtons », de façon flexible, avec d'importants retours en arrière. Il y a en effet une "capacité aussi à se remettre en cause. 
Tenter des choses mais assez vite de se rendre compte qu'on est en train d'aller au mur et de revenir » (E2). Le cas de l'expérience négative du premier DG l'illustre, tout comme celui de la tentative de scission du service commercial et du service achat: "on s'est aperçu trois mois après que ça paralysait en fait l'organisation de l'entreprise et que ça enlevait de l'initiative et qu'il y avait des choses que l'on ne voyait plus »(E2). On retrouve ici la capacité d'improvisation organisationnelle repérée par Julien (2001) chez les PME en forte croissance.

Pour autant, la présence d' "électrons libres » dans la structure reste caractéristique de l'entreprise. Par exemple, quand on demande au développeur de nouvelles filières quel est son poste dans l'entreprise, il indique : "Je n'ai pas de poste, je suis indépendant dans la boîte. Je ne veux pas de poste hiérarchisé, je veux être libre »(E3). Le fondateur lui-même le présente ainsi : il « est un entrepreneur. Ce n'est pas quelqu'un à qui vous allez dire: 'tu vas t'assoir à cette table et tu vas faire cela'. Il fait ce qu'il a envie de faire » (E1).

\subsection{5. Évolution des tensions paradoxales dans le temps}

Il est intéressant de noter l'apparition progressive de ces tensions paradoxales dans la trajectoire de croissance de BioDistri (cf. Figure 2). Ainsi, avant 1994, nous pouvons nous demander si la dynamique paradoxale avait réellement commencé. A ses débuts, BioDistri avait une vision clairement sociétale et son identité normative ne pouvait être mise en doute : "On était des baba-cool convaincus et on pensait qu'il fallait changer le monde. C'était vraiment ça, le projet de l'entreprise » (E1). L'entreprise a connu "une évolution très rapide, avec d'abord une croissance organique forte » (E2). Elle explorait et expérimentait de nouvelles façons de faire, sans structure organisationnelle définie et dans un certain désordre créateur (Thiétart et Forgues, 2006). : «On était très réactifs puisqu’on mangeait tous les midis ensemble à quatre autour d'une table. Donc on discutait de ça et puis une heure après, on commençait à mettre en place les choses » (E3).

\section{Figure 2 - Les tensions paradoxales dans la trajectoire de croissance de BioDistri}

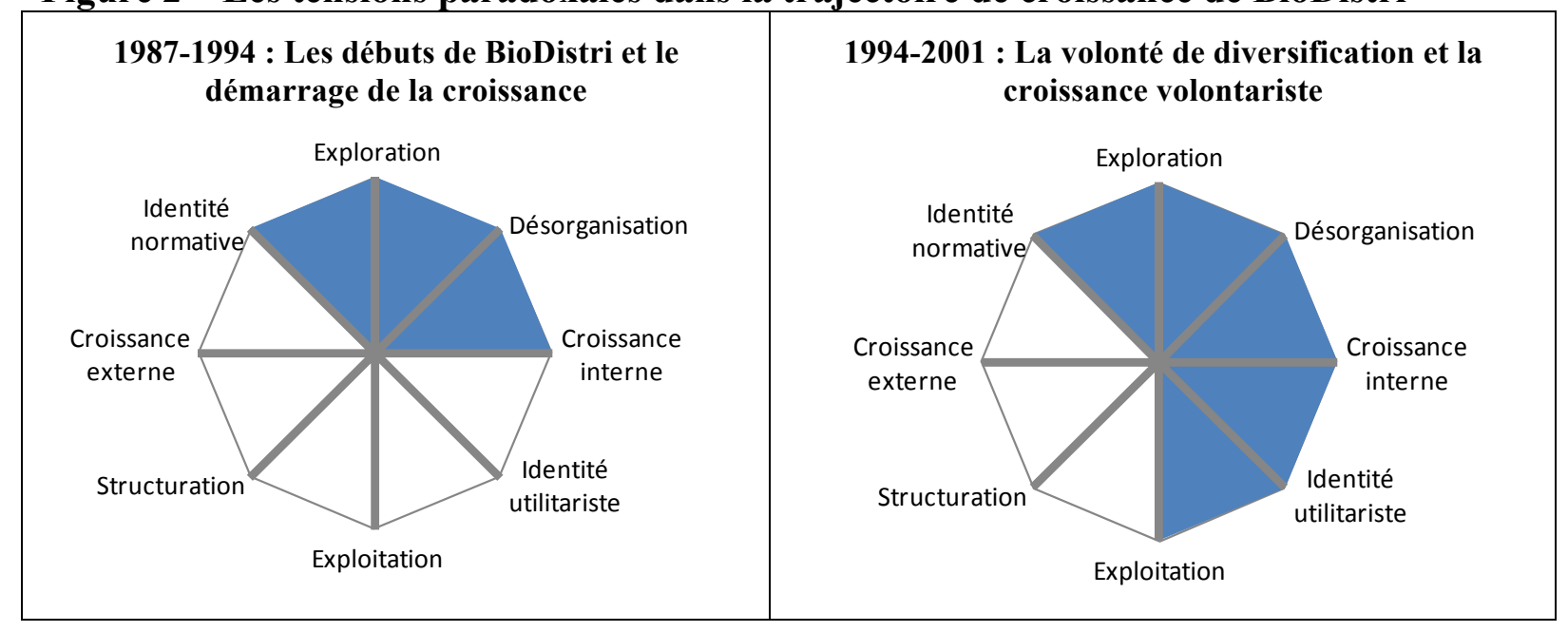




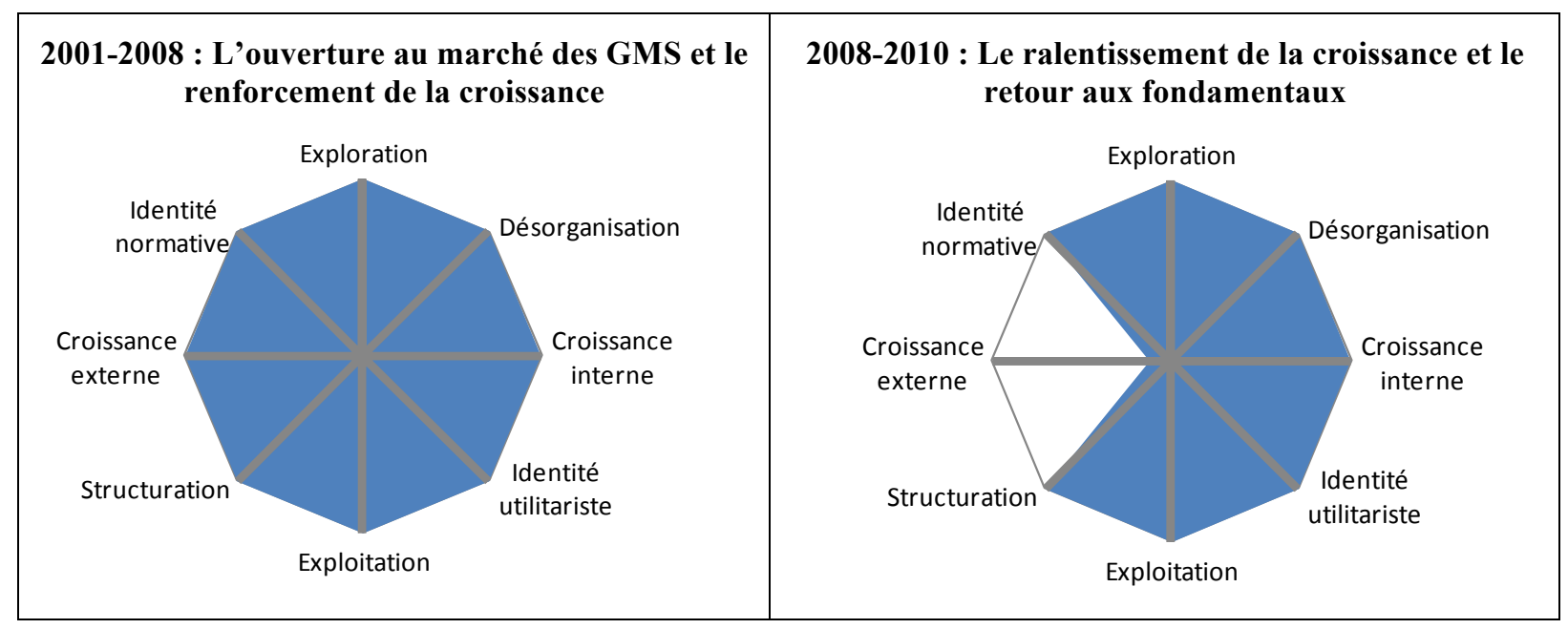

Pour autant, dès lors que ce pionnier du bio a fait le choix de la diversification et de la croissance volontariste, les seuls pôles "identité normative", "croissance interne", « exploration » et « désorganisation » ne lui ont plus été suffisants pour se développer : il a été nécessaire de s'appuyer simultanément sur leur contraire. Des tensions entre « exploration et exploitation » (poursuite des expérimentations, mais aussi recherche d'efficience au niveau logistique, qualité, etc.) et entre «identités normative et utilitariste » (bien que les valeurs sociétales soient conservées, les bénéfices puis la rentabilité deviennent un but prégnant) émergent alors relativement tôt dans sa trajectoire. Il faudra attendre une seconde phase de croissance soutenue, et plus précisément un virage stratégique avec l'ouverture au marché des GMS, pour percevoir celles existant entre "croissance interne et externe" (croissance organique et partenariats combinés à des acquisitions) et entre «désorganisation et structuration » (nécessaire structuration d'un désordre créateur).

A noter qu'à partir de 2008, avec le retour aux fondamentaux, la croissance se ralentit et redevient d'ailleurs avant tout une croissance organique, l'équipe dirigeante délaissant l'un des pôles de la tension " croissance interne et externe ». Rappelons que durant cette dernière période, la PME a fait le choix de privilégier son cœur de métier, en réaction à un environnement jugé hostile.

\subsection{Vers des logiques paradoxales de dialogue et de différenciation}

Quasi-absentes aux débuts, les tensions paradoxales se sont donc accentuées au fil de la trajectoire de croissance. En effet, durant les phases de forte croissance, les pôles initiaux ne suffisent plus à la PME pour se développer, qui s'appuie alors simultanément sur leur contraire. Pour faire face à ces tensions et à la lumière de la grille de lecture paradoxale développée par Josserand et Perret (2003), deux logiques semblent s'actionner chez BioDistri : une logique de dialogue et une logique de différenciation. Ces logiques reposent sur des pratiques associant deux dimensions : temporelle et spatiale. La dimension temporelle et spatiale évoque la nature de la dynamique managériale, à savoir plutôt de type dialectique dans une perspective de dialogue (prévoyant des espaces d'échanges et/ou des temps de rencontre) ou de type dichotomique dans une perspective de différenciation (prévoyant des espaces cloisonnés et/ou des temps successifs).

\subsubsection{Les pratiques favorisant la création de temps et d'espaces d'échanges dans une logique de dialogue}


L'analyse du cas a permis de dégager un ensemble de pratiques contribuant à créer des temps et espaces d'échanges favorables à la construction d'un équilibre entre les pôles en tension repérés au sein de la PME.

A l'interne, on note ainsi l'existence de pratiques qui contribuent à l'empowerment des salariés de BioDistri, avec en particulier des pratiques qui favorisent la responsabilisation et la participation dans la prise de décision, ainsi que l'échange d'information (Nauman, Khan et Ehsan, 2010; Seibert, Silver et Randolph, 2004). Ceci transparait tout d'abord dans le style de management plutôt participatif. En tout cas, le fondateur est décrit comme quelqu'un « qui est très proche des salariés, très apprécié des salariés, aimé des salariés » (E7), qui souhaite maintenir des échanges directs avec les salariés. Il se dit lui-même «très alimenté par les discussions avec tout le monde dans l'entreprise»(E1). Des réunions mensuelles programmées coexistent avec des temps d'échanges informels : "L'idée là est d'avoir une discussion totalement à bâtons rompus, ouverte, dans laquelle les gens peuvent, ou poser des questions, ou dire des choses » (E1). De plus, le fondateur n'est pas uniquement vu comme un personnage charismatique et visionnaire, mais aussi comme quelqu'un qui sait déléguer : " Il ne donne pas les clés à tout le monde mais il les a données à beaucoup de gens »(E3). La prise d'initiative est fortement valorisée au sein de BioDistri : "Donc c'est par exemple une des personnes qui a aussi joué un rôle moteur en allant trouver un nouveau paysan qui s'est installé, ou en disant 'tiens, le marché, c'est idiot parce qu'il n'y a pas de mangues aujourd'hui, si on en avait, on pourrait faire ça'. [...] Il y a plusieurs personnes dans l'entreprise qui ont eu ce rôle-là. »(E2). Dans cette perspective, du temps est dédié aux échanges pour promouvoir des idées, et la démocratisation de l'information joue un rôle crucial : "l'accès à l'information a toujours été quelque chose de très évident 》 (E2). Celle-ci s'appuie notamment sur des technologies de l'information et de la communication, telles que par exemple un «logiciel global» permettant de centraliser toute l'activité, un «système de tableaux croisés dynamiques » ouvert à tous, ou encore, un «Intranet qualité » qui fait partie intégrante du système qualité de la PME.

Au regard de la littérature, il peut être suggéré que les pratiques d'empowerment des salariés agissent plus particulièrement sur deux tensions paradoxales: "désorganisation et structuration » et «exploration et exploitation ». D'une part, bien que l'organisation se soit progressivement structurée, il reste néanmoins "une très grande latitude pour prendre des décisions au quotidien et c'est des décisions qui sont très engageantes »(E2). Comme le rappelle Julien (2001), une structure plus formalisée et complexe, mais en même temps une tendance à davantage déléguer et à adopter un management plus participatif, sont d'ailleurs caractéristiques des PME qui connaissent une forte croissance. D'autre part, la littérature suggère que l'empowerment soit un antécédent de la capacité des PME à innover en termes de produits et/ou process (Cakar et Ertürk, 2010). Il a notamment été montré que les styles de management participatifs, démocratiques ou collaboratifs encouragent l'innovation (Van de Ven, 1986), ou encore, que les PME innovantes seraient celles qui favorisent le partage des connaissances avec les salariés (Zhang, Macpherson et Jones, 2006). En particulier, l'empowerment aurait une forte influence non seulement sur l'exploration, mais aussi sur l'exploitation des connaissances (Lee et Lee, 2012).

A l'externe, les pratiques qui conduisent à multiplier les temps et espaces d'échanges concernent le développement d'un réseau social étendu et d'un véritable entrepreneuriat institutionnel. Premièrement, BioDistri crée et maintient des relations avec un grand nombre de parties prenantes du secteur, en France et à l'étranger, et a ainsi pu se doter d'un réseau social de plus en plus étendu, tant sur la scène nationale qu'internationale. Cela passe, d'une 
part, par des collaborations avec des organisations professionnelles du secteur, des agences régulatrices et des laboratoires d'analyse, afin d'échanger et d'être présent "dans tous les endroits où il faut veiller à la bonne qualité du travail en matière réglementaire, en matière de contrôle, de certification, etc. " (E1). D'autre part, son réseau s'est construit au fur et à mesure des rencontres avec des producteurs et distributeurs de fruits et légumes. Par exemple, BioDistri est allé à la rencontre «des gens qui faisaient de la banane en République Dominicaine », pour "regarder comment on pouvait travailler la banane »(E2). Comme l'affirme le Secrétaire général, il s'agit d'un «métier dans lequel certaines rencontres ont donné naissance à des projets importants» (E2). Deuxièmement, comme beaucoup d'entrepreneurs motivés par un développement plus durable (Shepherd et Patzelt, 2010), le fondateur de la PME se comporte en véritable entrepreneur institutionnel (Pacheco, York, Dean et Sarasvathy, 2010) au sein du secteur du bio. Dès les débuts, il a participé à la construction du marché et à l'invention d'un modèle économique basé sur une juste rémunération de l'ensemble des maillons de la chaîne. Il continue aujourd'hui à participer à la construction des normes et règles du secteur en étant " très investi auprès de la Commission Européenne, auprès du Ministère de l'Agriculture en France » (E1) et en occupant (lui-même ou la responsable qualité) des postes clés dans différentes institutions : organismes de certifications (Fair Trade, EcoCert), agence Bio, syndicat des transformateurs, comité bio de l'interfel (interprofession fruits et légumes), etc. De plus en plus, l'entreprise se veut un porteparole du bio en France auprès du grand public : mise en ligne d'un site informationnel, participations à différents salons et conférences, émissions radio et tv, etc.

Ces pratiques tournées vers l'externe, visant à développer un réseau social étendu et un entrepreneuriat institutionnel, ont ainsi permis à BioDistri de se doter d'un véritable capital social. Or, des études antérieures suggèrent que le capital social puisse faciliter l'acquisition de nouvelles connaissances et leur exploitation, en permettant la création de valeur à travers les échanges et la combinaison d'actifs intellectuels existants (Nahapiet et Ghoshal, 1998; YliRenko, Autio et Sapienza, 2001). Plus généralement, nombre de travaux ont étudié le rôle du capital social comme levier à l'exploration et l'exploitation des connaissances dans les PME (Thorpe, Holt, Macpherson et Pittaway, 2005). En d'autres termes, tant la création d'un réseau social étendu que le développement d'un entrepreneuriat institutionnel peut représenter une issue à la tension paradoxale " exploration et exploitation » à laquelle BioDistri fait face, en contribuant simultanément à ses deux pôles a priori opposés.

Par ailleurs, la volonté de développer un entrepreneuriat institutionnel est également un moyen de créer des interactions réciproques entre les pôles de la tension paradoxale « identités normative et utilitariste». En effet, l'implication de la PME dans différentes organisations et instances, en vue de contribuer à la construction des normes et règles du secteur, lui permet non seulement d'être garante d'une certaine éthique mais aussi de mener une véritable veille réglementaire et, ainsi, de prendre de l'avance sur les concurrents : " On a des concurrents qui ne sont membres d'aucune. C'est du temps, c'est aussi un investissement financier, mais on est informés des changements réglementaires »(E6). En outre, les différentes actions institutionnelles et communicationnelles menées dans le cadre de cet entrepreneuriat institutionnel permettent à BioDistri de se construire une réelle légitimité dans son secteur (Suchman, 1995) : "Quand on interroge les clients ou les prospects, [BioDistri] est un 'label' »(E2). De ce fait, la PME exploite les synergies existant entre les deux identités : la volonté de développer le marché bio constitue un avantage concurrentiel clé, la création de valeur sociétale étant aussi créatrice de valeur économique (Berger-Douce, 2011). Par exemple, le fort appui apporté aux producteurs tant sur le plan technique (conseil pour la certification et le choix des variétés, mise à disposition d'agronomes, etc.) que financier 
(avances sur récoltes, achat de matériel, etc.) correspond aussi à une demande de la part du consommateur qui «accepte de rémunérer toute cette chaîne, lui-même d'ailleurs trouvant des avantages en compensation du prix payé parce qu'il y trouve de la santé, du sens, de la responsabilité environnementale, sociale, etc.» (E1). Surtout, cela permet d'avoir un approvisionnement régulier, diversifié et de qualité, ce que les concurrents ne savent pas forcément faire dans un contexte d'offre inférieure à la demande : "la vraie différenciation, c'est vraiment sur la relation à l'amont. Quand on va rencontrer des clients ou des prospects, c'est vraiment, dans ce qu'on met en avant, ce qu'ils retiennent le plus »(E2).

En définitive, les pratiques mises en œuvre au sein de BioDistri dans une logique de dialogue, que ce soit à l'intérieur des frontières de l'entreprise (empowerment des salariés) ou davantage tournées vers l'externe (création d'un réseau social étendu et développement d'un entrepreneuriat institutionnel légitimant), contribuent à trouver une issue aux tensions paradoxales de BioDistri.

\subsubsection{Les pratiques de différenciation des profils et rôles}

Tout en favorisant le dialogue des contraires, BioDistri a aussi adopté une logique de différentiation des rôles et profils pour gérer les tensions paradoxales auxquelles elle fait face.

Pour croitre de manière conséquente et durable, BioDistri a dû acquérir des compétences, plus proches du monde des "Goliath", qui lui manquaient à ses débuts. Les propos du fondateur, quant à l'un des militants ayant contribué aux premiers développements de la PME, illustrent clairement ceci : "Je parle de lui parce que c'est un type de très grande qualité, c'est un très grand professionnel, mais il ne peut pas prendre la direction générale du groupe parce qu'il y a trop de choses qui lui manquent. Le directeur général, je suis obligé d'aller le chercher ailleurs... Et je vais le trouver chez des gens qui vont mettre un temps fou à s'adapter, à comprendre l'ensemble des rouages et la véritable manière de laquelle les problèmes arrivent dans une entreprise comme celle-ci» (E1). Ainsi, la PME a dû recruter des salariés au profil jugé plus «classique » que celui des militants du début, à savoir des diplômés d'écoles de commerce et/ou des individus ayant eu une expérience professionnelle dans des grandes entreprises. Également, BioDistri a intégré les pratiques et compétences des salariés des entreprises acquises au cours de ses opérations de croissance externe. Si certaines de ces entreprises étaient elles-mêmes pionnières sur le marché des produits biologiques et avaient une culture relativement similaire à celle de BioDistri, d'autres en revanche venaient d'un monde complètement différent, avec « une culture différente» (E7). Tel était le cas des entreprises acquises spécialisées dans la grande distribution.

Désormais, coexistent au sein de BioDistri les mondes des "David" militants et des "Goliath" plus classiques, chacun de ces deux mondes contribuant au développement de la PME tout en ayant des responsabilités bien distinctes. D'une part, le fondateur et les militants du début s'occupent essentiellement du développement des filières d'approvisionnement (suivi des agriculteurs, identification et développement de filières) et du travail institutionnel (lobbying, veille réglementaire, communication) au sein de la filière bio. Précisons que ces activités sont celles qui sont à la source des avantages concurrentiels que la PME détient sur ses concurrents plus imposants en taille : "La vraie différenciation, c'est vraiment les relations avec les fournisseurs» (E2) ; "La plupart de nos concurrents ne sont pas impliqués dans les institutions de la filière biologique, et donc ils ne sont pas rapidement informés de tout changement, contrairement à nous » (E1). Ils sont aussi en charge du marché des magasins spécialisés, étant donné que celui-ci comprend des distributeurs souvent eux-mêmes militants. 
D'autre part, les cadres à profil dit plus «classique » sont responsables de la logistique et des activités commerciales sur le marché de la grande distribution. Ils gèrent également les relations avec les partenaires financiers, ainsi que les opérations de croissance externe (l'intégration des entreprises acquises). Ce constat révèle clairement que la différenciation des rôles a été un moyen pour BioDistri de gérer les tensions paradoxales relatives à la présence simultanée d'opérations de croissance interne et externe, et d'une identité normative et utilitariste. La DRH précise en effet qu'il est difficile «d'arriver à conjuguer les deux milieux. Le milieu de la finance de partenaires et puis du staff de la direction qui vient de grandes écoles et puis qui a une formation de 15 ou 20 premières années quelque part dans un grand groupe et puis [ceux] qui sont vraiment des hommes de terrain... D'arriver à conjuguer tout ça c'est compliqué, parce qu'on est dans une toute petite boîte, les uns avec les autres »(E7).

Ce constat rejoint celui de Battilana et Dorado (2010), qui montrent que le développement des entreprises hybrides passe par la création d'une identité organisationnelle commune permettant un équilibre entre les logiques, et ce, via les leviers du recrutement et de la socialisation (formation, communication interne, promotion, système incitatif, etc.). Parmi les deux modèles repérés par les auteurs, BioDistri s'apparente à l'approche intégration qui combine recrutement "mix-and-match » (à partir des capacités individuelles à mettre en œuvre la logique commerciale ou sociale) et approche de la socialisation centrée sur les finalités. Or, si cette approche permet la plus forte croissance, elle serait davantage source de tensions entre sous-groupes que l'approche apprentissage qui combine recrutement « tabula rasa » (à partir des capacités d'adaptation à l'autre logique) et approche de la socialisation centrée sur les moyens.

Bien que cette logique de différenciation des profils et des rôles ait été privilégiée jusqu'ici, on constate qu'elle laisse place progressivement à une logique de dialogue au moyen d'une organisation en mode projet, comme le relate le fondateur au sujet de la filière belge : " On est en train de travailler en mode projet avec des gens des deux camps et qui, tout à coup, découvrent qu'ils ont vraiment des choses utiles à faire ensemble, et qu'ils sont contents de travailler ensemble. Peut-être que là, on commence à trouver un modèle qui va être un modèle de terrain commun. Ce que je crois, moi, intéressant. Je pense que si on arrive à cela, on aura énormément de force » (E1).

\section{Discussion et conclusion}

Dans la lignée des travaux qui suggèrent que la réussite des stratégies d'hypercroissance des PME repose sur la gestion combinée de couples dits paradoxaux (Chanut-Guieu et Guieu, 2011b), la présente recherche vise à analyser les tensions paradoxales qui parcourent la trajectoire d'une PME à forte croissance, ainsi que les pratiques mises en œuvre pour y faire face. Elle se centre sur le contexte particulier des PME axées sur la durabilité, un contexte quelque peu négligé dans la littérature sur la croissance (Nazarkina, 2012).

L'analyse du cas de BioDistri a permis d'identifier quatre tensions paradoxales majeures qui sont apparues au cours de sa trajectoire de croissance : (1) identités normative et utilitariste ; (2) exploration et exploitation ; (3) croissance interne et externe ; (4) désorganisation et structuration. Précisons que les trois dernières tensions sont sans doute présentes dans toute PME en forte croissance. Par exemple, Chanut-Guieu et Guieu (2011b) repèrent, dans leur étude sur les PME en hypercroissance, les tensions «exploration/exploitation» et 
« croissance interne/croissance externe ». La tension « désorganisation/structuration » a, quant à elle, été déjà discutée dans nombre de travaux antérieurs sur les PME en forte croissance. Notamment, pour Julien (2001), ces entreprises auraient la capacité à gérer le désordre, tout en adoptant une structure plus formalisée. En revanche, la première tension, celle intitulée " identité normative/identité utilitariste», semble être davantage spécifique aux PME axées sur la durabilité, voire plus généralement aux entreprises dites hybrides (Battilana et Dorado, 2010; Pache et Santos, 2010). Finalement, l'ensemble des tensions paradoxales repérées chez BioDistri traduit la coexistence, caractéristique des "high-growth David", de deux mondes opposés : ceux des "David" militants et des "Goliath". En effet, cette PME axée sur la durabilité serait parvenue à croitre rapidement en combinant le meilleur de ces deux mondes, en articulant notamment l'innovation de produits et la bonne compréhension du marché bio des "David" militants, avec l'innovation de processus et le management plus professionnel des "Goliath" (Hockerts et Wüstenhagen, 2010).

Par ailleurs, l'analyse de l'évolution dans le temps de ces tensions révèle qu'elles se sont renforcées au cours de la trajectoire de croissance de la PME, montrant le lien fort qu'il existe entre tensions paradoxales et forte croissance. Ce constat rejoint celui avancé par ChanutGuieu et Guieu (2011a), qui évoquent une relation dynamique entre l'hypercroissance et les paradoxes. Toujours est-il que la littérature suggère que les paradoxes peuvent être tour à tour vécus comme un moteur ou un frein à l'action (Giordano, 2003) : d'un côté, ils représentent une occasion de changement, pour l'individu comme pour l'organisation, en encourageant une dynamique créative ; d'un autre côté, ils sont générateurs de stress et de fatigue professionnelle en positionnant l'individu dans une situation difficile à résoudre, parfois vécue comme un enfermement dans une contradiction sans solution apparente.

La présente recherche montre alors que, dans le cas étudié, les situations paradoxales ont trouvé leur équilibre dans des logiques à la fois de dialogue et de différenciation (Josserand et Perret, 2003). Des clés stratégiques ont ainsi été repérées pour faire face aux tensions paradoxales, au travers des pratiques mises en œuvre par la PME, en ce sens qu'elles ont permis à l'équipe dirigeante d'ouvrir certains paradoxes et d'en exploiter la richesse, plutôt que de s'enfermer dans une dualité sclérosante (Barel, 1989; Bernard-Weil, 2002). D'une part, dans une logique de dialogue, celles-ci englobent plus particulièrement des pratiques d'empowerment des salariés, mais aussi des pratiques qui repoussent les frontières de l'organisation en développant un réseau social étendu et un entrepreneuriat institutionnel légitimant. D'autre part, cette logique de dialogue cohabite avec une logique de différenciation. En effet, si les profils et compétences au sein de BioDistri traduisent la coexistence de deux mondes a priori antagonistes (ceux des "David" militants et des "Goliath"), force est de constater qu'un certain travail de segmentation a été effectué pour éviter les frictions et limiter les points de rencontres entre ces mondes distants. Par exemple, les opérations de croissance externe sont gérées par des cadres à profil " classique », et non pas par les pionniers qui se consacrent, quant à eux, principalement aux activités à la source des avantages concurrentiels de la PME. Très certainement, cette logique de différenciation est inhérente au fait que la PME étudiée soit axée sur la durabilité. Plus généralement, une telle logique tend effectivement à être adoptée par les entreprises dites hybrides (Battilana et Dorado, 2010). Toutefois, chez BioDistri, cette logique de différenciation semble progressivement laisser place à une logique de dialogue, avec notamment la volonté de plus en plus prégnante de s'organiser en mode projet : la logique de différenciation pourrait donc ne constituer qu'une étape intermédiaire vers celle de dialogue déjà initiée.

En définitive, et comme le soulignent Guedri, Hussler et Loubaresse (2014, p. 13), «manager 
les tensions coopétitives, [...] gérer les contradictions, dépasser les paradoxes 》 sont " autant d'injonctions qui jalonnent le quotidien des managers et des organisations ». Dès lors, l'approche par les paradoxes utilisée dans le présent article peut être un outil de caractérisation de situations complexes, utile tant pour les chercheurs que pour les praticiens faisant face à de telles situations. En outre, cette recherche permet d'aller au-delà de la seule identification des tensions paradoxales. En effet, si leur identification par les dirigeants de PME axées sur la durabilité et/ou à forte croissance est indispensable au développement d'une capacité à la gestion paradoxale, elle n'est pas suffisante : une réflexion sur sa mise en œuvre est également nécessaire (Josserand et Perret, 2003). Des stratégies doivent donc être pensées pour gérer ces tensions (Hahn, Pinkse, Preuss et Figge, 2015). Toujours est-il que les contributions antérieures se sont principalement limitées à repérer les différents types de paradoxes présents dans les organisations (Perret et Josserand, 2003; Smith et Lewis, 2011), sans réellement se pencher sur leurs modes de gestion ou sur les dynamiques managériales et organisationnelles afférentes. Cette recherche contribue ainsi non seulement à enrichir la compréhension des tensions paradoxales inhérentes aux PME durables en forte croissance mais aussi, et surtout, à questionner les pratiques mises en œuvre pour y faire face. Sa contribution réside donc principalement dans la proposition de « clés stratégiques », en vue de sensibiliser à leurs enjeux les dirigeants de PME qui cherchent à se développer, et ce, d'autant plus dès lors que la PME en question est qualifiée d'hybride. Notons que ces clés stratégiques, résumées dans la figure ci-après (cf. Figure 3), peuvent être associées plus ou moins directement à certaines tensions paradoxales. Toutefois, il ne s'agit pas, ici, de construire une boite à outils pour gérer les paradoxes mais de dégager des pratiques ouvrant la voie à une possible équilibration du système.

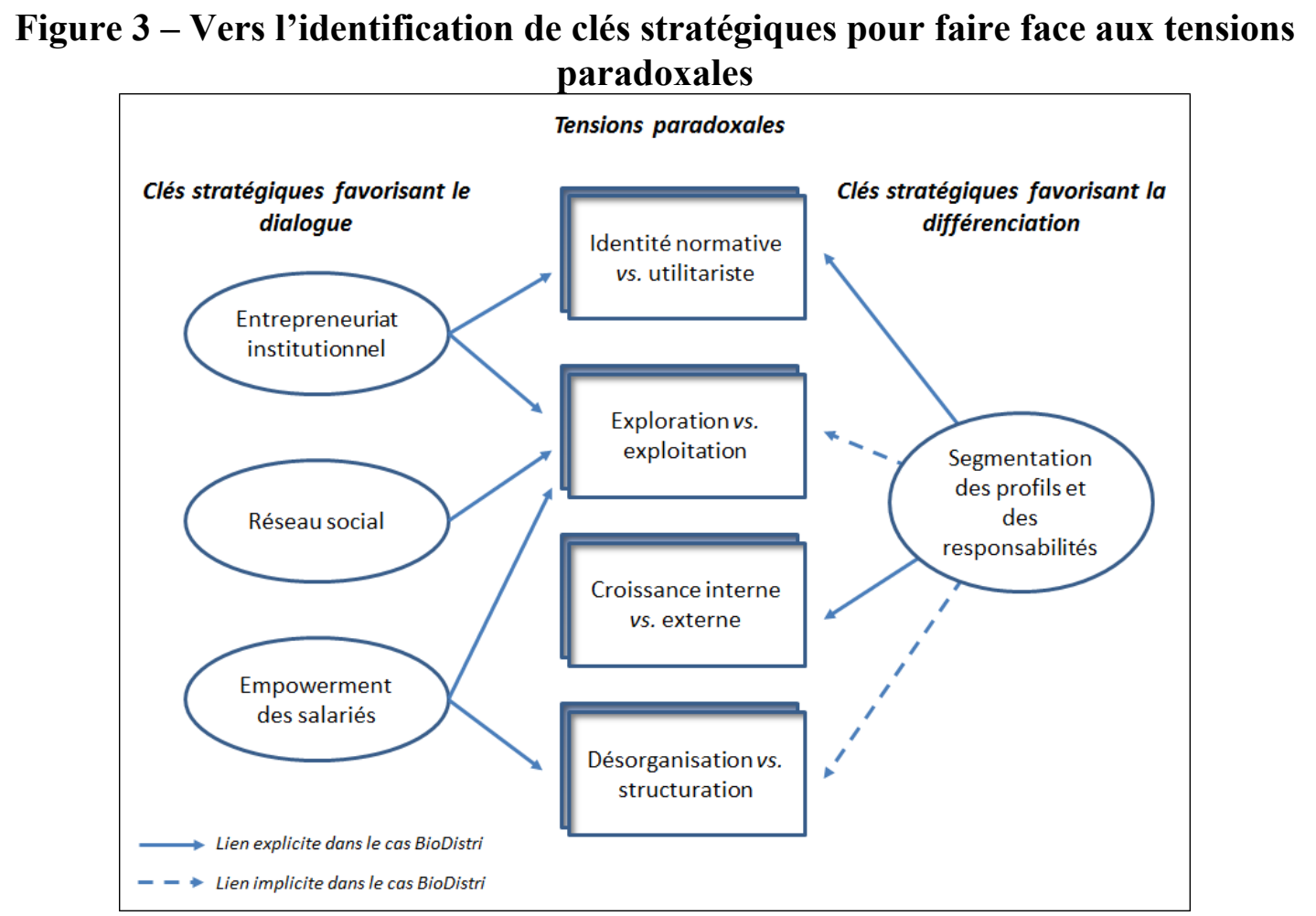

Cette recherche n'est toutefois pas exempte de limites d'ordre méthodologique et analytique. Premièrement, il serait intéressant d'analyser en profondeur la phase de ralentissement de croissance de BioDistri. Cette période se caractérise plus particulièrement par un retour aux fondamentaux: en réaction à un environnement hostile (les clients se tournent vers les nouveaux entrants et plusieurs grossistes bio rencontrent des difficultés), la PME a fait le 
choix de privilégier son « cœur de métier ». On peut alors s'interroger sur les conséquences de ce recentrage sur les tensions paradoxales. En effet, à ce stade-ci, on constate que l'équipe dirigeante fait le choix de trancher en faveur de l'un des pôles de la tension « croissance interne et externe $»$ : tout en poursuivant la mise en place de partenariats, elle prend surtout partie pour le pôle « croissance interne ». Il est toutefois difficile de pousser plus en avant ce constat, étant donné que seules les deux premières années de cette phase ont été couvertes par la collecte de données. Deuxièmement, si la gestion de multiples tensions paradoxales est l'une des conditions requises pour instaurer une dynamique d'hypercroissance, elle n'est pas suffisante : l'existence d'une logique dominante s'avère également nécessaire pour canaliser les déséquilibres sous-jacents (Chanut-Guieu et Tannery, 2009). Ainsi, il conviendrait d'intégrer une réflexion sur cette notion de logique dominante, dans une étude subséquente portant sur les tensions paradoxales au sein des PME axées sur la durabilité et à forte croissance. Troisièmement, cette recherche a repéré une tension relevant de la mise en pratique de valeurs a priori opposées. Il serait donc intéressant d'approfondir les enjeux de la diversité de valeurs (Jehn, Northcraft et Neale, 1999), au sein des équipes de direction des PME qui combinent les mondes des "David" militants et des "Goliath" pour croître. En effet, bien que de nombreux travaux se soient intéressés à l'effet de la diversité sur la croissance des entreprises, leurs résultats empiriques restent aujourd'hui encore équivoques (Buyl, Boone, Hendricks et Matthyssens, 2011). Quatrièmement, rappelons que cette étude porte sur un cas unique, ce qui tend à limiter la généralisation possible des résultats, et que les paradoxes identifiés ont émergé du discours des répondants. Cette recherche n'a, de ce fait, pas la prétention d'en dresser une liste exhaustive. Le concept de paradoxe, exploité ici dans sa capacité heuristique à faire émerger du sens, a néanmoins permis de questionner un phénomène jugé complexe.

\section{Références bibliographiques}

Alter, N. (2002). Théorie du don et sociologie du monde du travail. Revue du MAUSS, 20, 263-285.

BAREL, Y. (1989). Le paradoxe et le système. Grenoble, Presses Universitaires de Grenoble.

BATtilana, J. et Dorado, S. (2010). Building sustainable hybrid organizations: the case of commercial microfinance organizations. Academy of Management Journal, 53(6), 1419-1440.

Berger-Douce, S. (2005). Management environnemental et PME : apports et limites d'une démarche collective. Revue Internationale P.M.E., 18(3-4), 93-123.

BERgER-Douce, S. (2011). Le développement durable, un levier d'innovation pour les PME ? Revue Française de Gestion, 6(215), 147-166.

BERNARD-WeIL, E. (2002). Stratégies paradoxales en biomédecine et sciences humaines. Paris, L'Harmattan.

BIERLY, P.E. et DALY, P.S. (2007). Alternative knowledge strategies, competitive environment, and organizational performance in small manufacturing firms. Entrepreneurship Theory and Practice, July, 493-516.

Bonneveux, E., Calmé, I. et Soparnot, R. (2012). Strategic CSR assets within SMEs: a comparative case study. International Business Research, 5(7), 38-49.

Buyl, T., Boone, C., Hendricks, W. et Matthyssens, P. (2011). Top management team functional diversity and firm performance: the moderating role of CEO characteristics. Journal of Management Studies, 48(1), 151-177. 
CAKAR, N.D. et ERTÜRK, A. (2010). Comparing innovation capability of small and mediumsized enterprises: examining the effects of organizational culture and empowerment. Journal of Small Business Management, 48(3), 325-359.

Chanal, V. et Mothe, C. (2005). Concilier innovations d'exploitation et d'exploration. Le cas du secteur automobile. Revue Française de Gestion, 31(154), 173-191.

Chanut-Guieu, C. et Guieu, G. (2011a). Stratégie et structuration des trajectoires d'hypercroissance des PME. Une étude de cas comparative. Management \& Avenir, 3(43), $37-56$.

Chanut-Guieu, C. et Guieu, G. (2011b). PME en hypercroissance : quelles clés de succès ? Business Management Review, 1(2), 24-35.

Chanut-Guieu, C. et TAnnery, F. (2009). La stratégie d'hypercroissance des moyennes entreprises. Entre logique dominante et pratiques stratégiques paradoxales. Actes de la

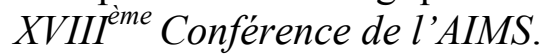

Chanut-Guieu, O., Guieu, G., Tannery, F. et Dana, L-P. (2014). Au-delà de la croissance rapide : combiner le paradoxe et la logique dominant pour un modèle d'hypercroissance durable. $12^{\text {ème }}$ congrès international francophone en entrepreunariat et PME, Octobre, Agadir.

ChOI, D.Y. et GRAY, E.R. (2008). The venture development processes of sustainable entrepreneurs. Management Research News, 31(8), 558-569.

Courrent, J-M. (2012). RSE et développement durable en PME. Bruxelles, De Boeck Supérieur.

De Colle, S., Henriques, A. et Sarasvathy, S. (2014). The paradox of corporate social responsibility standards. Journal of Business Ethics, 125, 177-191.

EISENHARDT, K.M. (2000). Paradox, spirals, ambivalence: the new language of change and pluralism. Academy of Management Review, 25(4), 703-705.

Fassin, Y., Rossem, A.V. et Buelens, M. (2011). Small-business owner-managers' perceptions of business ethics and CSR-related concepts. Journal of Business Ethics, 98(3), 425-453.

Fayard, P. et Blondeau, E. (2014). La force du paradoxe. En faire une stratégie. Paris, Dunod.

Fisher, E., Reuber, A.R., Habadou, M., Johnson, W. et Lee, S. (1997). The role of socially constructed temporal perspectives in the emergence of rapid growth firms. Entrepreneurship, Theory and Practice, 22(2), 13-30.

Fombrun, C.J. et Wally, S. (1989). Structuring small firms for rapid growth. Journal of Business Venturing, 4(2), 107-122.

Giordano, Y. (2003). Les paradoxes : une perspective communicationnelle. Dans V. Perret et E. Josserand (dir.), Le paradoxe, penser et gérer autrement les organisations (p. 115-128). Paris, Ellipses.

GRIMAND, A. (2013). La gestion des ressources humaines dans les PME en hypercroissance: frein ou levier? Revue Internationale P.M.E., 26(3/4), 89-115.

Grimand, A., Vandangeon-Derumez, I. et Schafer, P. (2014). Manager les paradoxes de la RSE. Revue Française de Gestion, 240, 133-148. 
Guedri, Z., Hussler, C. et Loubaresse, E. (2014). Contradictions, paradoxes et tensions en contexte(s). Revue Française de Gestion, 240, 13-28.

Hahn, T., Pinkse, J., Preuss, L. et Figge, F. (2015). Tensions in corporate sustainability: towards an integrative framework. Journal of Business Ethics, 127, 297-316.

Hahn, T., Preuss, L., Pinkse, J. et Figge, F. (2015).Cognitive frames in corporate sustainability: managerial sensemaking with paradoxical and business case frames. Academy of Management Review, 4015(1), 18-42.

Hall, J.K., DaneKe, G.A. et Lenox, M.J. (2010). Sustainable development and entrepreneurship: past contributions and future directions. Journal of Business Venturing, 25(5), 439-448.

Hockerts, K. et WÜstenhagen, R (2010). Greening Goliaths versus emerging Davids Theorizing about the role of incumbents and new entrants in sustainable entrepreneurship. Journal of Business Venturing, 25(5), 481-492.

JAY, J. (2013). Navigating paradox as a mechanism of change and innovation in hybrid organizations. Academy of Management Journal, 56(1), 137-159.

Jehn, K.A., NorthCraft, G.B. et Neale, M.A. (1999). Why differences make a difference: a field study of diversity, conflict, and performance in workgroups. Administrative Science Quarterly, 44(4), 741-763.

JENKINS, H. (2009). A 'business opportunity' model of corporate social responsibility for small- and medium-sized enterprises. Business Ethics: A European Review, 18(1), 21-36.

Josserand, E. et Perret, V. (2003). Pratiques organisationnelles du paradoxe. Dans V. Perret et E. Josserand (dir.), Le paradoxe, penser et gérer autrement les organisations (p. 165-187). Paris, Ellipses.

Julien, P.A. (2001). Les PME à forte croissance et la métaphore du jazz. Comment gérer l'improvisation organisationnelle de façon cohérente. Revue Internationale P.M.E., 14(3/4), 129-162.

KeChiche, A. et Soparnot, R. (2012). CSR within SMEs: literature review. International Business Research, 5(7), 97-104.

LEE, D.S. et LEE, K.C. (2012). Exploitation and exploration as links between psychological empowerment and creativity. Lecture Notes in Electrical Engineering, 181, 213-220.

LEWIS, M.W. (2000). Exploring paradox: toward a more comprehensive guide. Academy of Management Review, 25(4), 760-776.

MARCH, J.G. (1991). Exploration and exploitation in organizational learning. Organization Science, 2, 71-87.

Martinet, A.-C. (1990). Épistémologie de la stratégie. Dans A.-C. Martinet (coord.), Épistémologies et sciences de gestion (p. 211-236). Paris, Economica.

Miles, B.M. et Huberman, A.M. (2003). Analyse des données qualitatives. Paris, De Boeck.

Moore, G. et Spence, L. (2006). Editorial: responsibility and small business. Journal of Business Ethics, 67, 219-226.

Moreno, A.M. et CASILlas, J.C. (2007). High-growth SMEs versus non-high-growth SMEs: a discriminant analysis. Entrepreneurship \& Regional Development, 19, 69-88. 
Moss, T.W., Short, J.C., PAyne, G.T. et Lumpkin, G.T. (2011). Dual identities in social ventures: an exploratory study. Entrepreneurship Theory and Practice, 35(4), 805-830.

Mustar, P. (2002). Les PME à forte croissance et l'emploi. Paris, OCDE.

NAHAPIET, J. et GHOShaL, S. (1998). Social capital, intellectual capital and the organizational advantage. Academy of Management Review, 23, 242-266.

Nauman, S., Khan, A.M. et EhSAn, N. (2010). Patterns of empowerment and leadership style in project environment. International Journal of Project Management, 28, 638-649.

NAZARKINA, L. (2012). How to grow your organic business: multiple organizational identities of sustainable enterprises and their growth strategies. GRONEN Research Conference.

NiCHOLLS-NiXON, C.L. (2005). Rapid growth and high performance: the entrepreneur's impossible dream? Academy of Management Executive, 19(1), 77-89.

Pache, A.-C. et SAntos, F. (2010). Inside the hybrid organization: Selective coupling as a response to conflicting institutional logics. Academy of Management Journal, 35(3), 455-476.

Pacheco, D., York, J., Dean, T. et Sarasvathy, S. (2010). The co-evolution of institutional entrepreneurship: a tale of two theories. Journal of Management, 36, 974-1010.

PARADAS, A. (2011). Développement durable en petites entreprises. De la sensibilisation à l'engagement. La Revue des Sciences de Gestion, 247-248, 129-137.

PARRISH, B.D. (2010). Sustainability-driven entrepreneurship: principles of organization design. Journal of Business Venturing, 25(5), 510-523.

Perret, V. et Josserand, E. (DIR.) (2003). Le paradoxe : penser et gérer autrement les organisations. Paris, Ellipses.

Rouleau, L., Allard-Poesi, F. et Warnier, V. (2007). Le management stratégique en pratiques. Revue Française de Gestion, 33(174), 15-24.

SEIBERT, S.E., Silver, S.R. et RANDOLPH, W.A. (2004). Taking empowerment to the next level: a multiple-level model of empowerment, performance, and satisfaction. Academy of Management Journal, 47(3), 332-349.

SÉVILle, M. et WiRTZ, P. (2010). Caractéristiques et dynamique de l'équipe dirigeante dans une jeune entreprise en hypercroissance. Revue Internationale P.M.E., 23(3/4), 43-70.

ShePherd, D.A. et PAtZelt, H. (2010). The new field of sustainable entrepreneurship: studying entrepreneurial action linking 'what is to be sustained' with 'what is to be developed'. Entrepreneurship Theory and Practice, 35(1), 137-163.

SMith, W.K. et LEWIS, M.W. (2011). Toward a theory of paradox: a dynamic equilibrium model of organizing. Academy of Management Review, 36(2), 381-403.

Smith, W.K., Gonin, M. et Besharov, M.L. (2013). Managing social-business tensions: a review and research agenda for social enterprise. Business Ethics Quarterly, 23(3), 407-442.

Stewart, H. et GAPP, R. (2014). Achieving effective sustainable management: a smallmedium enterprise case study. Corporate Social Responsibility and Environmental Management, 21, 52-64.

Suchman, M. (1995). Managing legitimacy: strategic and institutional approaches. Academy of Management Review, 20(3), 571-610.

Thiétart, R.A. et Forgues, B. (2006). La dialectique de l'ordre et du chaos dans les organisations. Revue Française de Gestion, 32, 47-66. 
Thorpe, R., Holt, R., Macpherson, A. et Pittaway, L. (2005). Using knowledge within small and medium-sized firms: a systematic review of the evidence. International Journal of Management Reviews, 7(4), 257-281.

VAN de Ven, A.H. (1986). Central problems in the management of innovation. Management Science, 32, 509-607.

WACHeuX, F. (1996). Méthodes qualitatives et recherche en gestion. Paris, Economica.

WeInBERG, A. (1998). Distinguishing among green businesses: growth, green and anomie. Society \& Natural Resources, 11(3), 241-250.

YIN, R.K. (1994). Case study research. Design and methods. Londres, Sage Publications.

Yli-Renko, H., Autio, E. et SAPIenza, H.J. (2001). Social capital, knowledge acquisition, and knowledge exploitation in young technology-based firms. Strategic Management Journal, $22,587-613$.

Zahra, S.A., Gedajlovic, E., Neubaum, D.O. et Shulman, J.M. (2009). A typology of social entrepreneurs: motives, search processes and ethical challenges. Journal of Business Venturing, 24(5), 519-532.

Zhang, M., MACPHERSON, A. et JONES, O. (2006). Conceptualizing the learning process in SMEs: improving innovation through external orientation. International Small Business Journal, 24(3), 299-323. 